MATHEMATICS OF COMPUTATION

Volume 69, Number 230, Pages 547-581

S 0025-5718(99)01087-X

Article electronically published on March 3, 1999

\title{
ADAPTIVE MULTILEVEL METHODS IN SPACE AND TIME FOR PARABOLIC PROBLEMS-THE PERIODIC CASE
}

\author{
J. B. BURIE AND M. MARION
}

\begin{abstract}
The aim of this paper is to display numerical results that show the interest of some multilevel methods for problems of parabolic type. These schemes are based on multilevel spatial splittings and the use of different time steps for the various spatial components.

The spatial discretization we investigate is of spectral Fourier type, so the approximate solution naturally splits into the sum of a low frequency component and a high frequency one. The time discretization is of implicit/explicit Euler type for each spatial component.

Based on a posteriori estimates, we introduce adaptive one-level and multilevel algorithms.

Two problems are considered: the heat equation and a nonlinear problem.

Numerical experiments are conducted for both problems using the one-level and the multilevel algorithms. The multilevel method is up to $70 \%$ faster than the one-level method.
\end{abstract}

\section{INTRODUCTION}

The aim of this paper is to present a posteriori error estimates and numerical results that show the interest of some multilevel techniques for problems of parabolic type. Our methods are based on multilevel spatial splittings and the use of different time steps for the various spatial components.

The spatial discretization we consider is of Fourier type. Such a discretization allows one to simply define a two-level spatial decomposition; but, the authors believe that the general idea of the multilevel method extends to other types of spatial discretization and, in particular, to finite element methods (see Marion and $\mathrm{Xu} 11$ for a first step in that direction, based on $L^{2}$ orthogonal decomposition).

Let $T>0$. To compute an approximate solution of the problem on the timeinterval $(0, T)$, we first design an adaptive classical (one-level) method. It is based on an a posteriori analysis inspired by the work of C. Johnson and his coworkers 5, 6, 7, 10, for finite element methods.

For time advancing, we use a variant of the discontinuous Galerkin method of order 0 (see Eriksson, Johnson and Thomée [8]), in which the nonlinear term is treated explicitly. It can also be viewed as a variant of the implicit/explicit Euler scheme where the force function is integrated over time exactly.

Received by the editor October 15, 1997 and, in revised form, April 14, 1998.

1991 Mathematics Subject Classification. Primary 65M15, 65M70, 65B99.

Key words and phrases. Multilevel methods, Fourier Galerkin, parabolic equations, a posteriori error estimates, adaptive algorithms. 
Let $k_{n}$ be the time-step at iteration $n$, and $t_{n}=\sum_{i=1}^{n} k_{i}$. Denote by $S_{M_{n}}$ the space of trigonometric polynomials of degree less than or equal to $M_{n} / 2$ in each variable. The solution is spatially approximated at time $t_{n}$ by $U_{n} \in S_{M_{n}}$. At every iteration $n$, the adaptive method finds the discretization parameters $k_{n}$ and $M_{n}$ such that an appropriate norm of the error is below a given tolerance.

The a posteriori error analysis and the adaptive algorithm are investigated for two problems: a linear one (heat equation) and a nonlinear one. For nonlinear problems, it is well known that the approach developed by Johnson and his coworkers leads to a posteriori estimates involving stability constants that are quite delicate to compute (see [10] for a discussion of this question). This difficulty occurs here of course, but it is not at all related to the possible introduction of a multilevel strategy - the a posteriori estimates in the one-level and multilevel cases will involve the same constants. Therefore, throughout this paper we choose to deal with some model nonlinear equations for which the stability constants can be evaluated analytically; these equations have some common features with the 2D Navier-Stokes equations. Our analysis of the one-level (and multilevel) algorithm extends easily to these equations, as well as to many other nonlinear problems.

Next, we consider multilevel decompositions. For each $n \geq 1$, let $m_{n}$ and $M_{n}$ be two integers such that $1<m_{n} \leq M_{n}$. The two-level spatial decomposition of $S_{M_{n}}$ can be written as

$$
S_{M_{n}}=S_{m_{n}} \oplus\left(P_{M_{n}}-P_{m_{n}}\right) S_{M_{n}} .
$$

Based on this splitting, we look for an approximate solution in the form

$$
U_{n}=V_{n}+W_{n}, \text { where } V_{n} \in S_{m_{n}}, W_{n} \in\left(P_{M_{n}}-P_{m_{n}}\right) S_{M_{n}} .
$$

The component $V$ is made up of the lower modes of $U$, whereas $W$ is made up of the higher ones. Again the temporal discretization for each of these components is a variant of the discontinuous Galerkin method of order 0. But, $V$ is integrated with a time step $k$, whereas $W$ is integrated with a time step $K>k$. Equations for $V$ and $W$ are coupled through the previous set of modes of $U$ and the (possible) nonlinear term (see Sections 2.2 and 3.2 for a detailed definition of the multilevel scheme). We derive an a posteriori error estimate for our scheme. Then, we design an adaptive algorithm. For a nonnegative integer $p$ given by the user, at every iteration $n$, the algorithm finds discretization parameters $k_{n}, m_{n}$ and $M_{n}$, and $K_{n}$ is set equal to $p k_{n}$, so that the error is below a given tolerance.

Let us now motivate this multilevel strategy. For 2D Navier-Stokes equations (see Foias, Manley and Temam [9]), as well as for many other parabolic problems, it can be shown that the energy carried in the higher modes of the solution is much smaller than that carried in the lower modes. Consequently, the contribution to the error of the higher modes should be small. Therefore, it seems natural to integrate them with a larger time step and hope not to spoil the overall accuracy. This does not contradict the fact that the higher modes may evolve faster than the lower ones; nor the fact that the stability of the scheme may require a smaller time step for the higher modes, since we only aim to compute the solution with some given accuracy.

Moreover, the interest of the multilevel strategy has been shown in Burie \& Marion [2], thanks to a stability analysis and a priori error estimates for a nonadaptive version of the multilevel method; so have earlier computational studies for the 2D Navier-Stokes equations in Debussche, Dubois and Temam [4]. Nevertheless, in 
4], no a posteriori analysis is carried out and therefore the adaptive criteria are different; also the numerical performances of the algorithm are not compared to those of a 'classical' (one-level) adaptive method.

For both problems, our numerical results show the stability of the method and the interest of adaptivity. In computing time, the multilevel method is clearly superior; we have a gain of up to $70 \%$. Moreover, we observe that the smaller the tolerance is, the more important the gain in computing time is.

The paper is organized as follows. Section 2 focuses on the linear problem. Both, for the one-level and the multilevel methods, we give an a posteriori error estimate and introduce an adaptive algorithm. Then, we discuss some implementation issues and present some numerical tests. Section 3 adresses similar questions for the nonlinear equation. The theoretical results are only presented in these sections. Since the corresponding proofs are long and technical, we only give the main steps in the Appendix in the (more difficult) case of the nonlinear equations. The reader is referred to Burie [1] for more details concerning the proofs.

\section{A linear PRoblem}

Let $\Omega=(0,2 \pi)^{2}$ and $T>0$. In this section, we consider the following heat equation.

Find $u: \Omega \times[0, T] \rightarrow \mathbb{R}^{2}$ such that

$$
\left\{\begin{array}{l}
\frac{\partial u}{\partial t}(x, t)-\Delta u(x, t)=f(x, t), \forall(x, t) \in \Omega \times(0, T), \\
u(., t) \text { is } \Omega \text {-periodic, } \forall t \in(0, T), \\
u(x, 0)=u_{0}(x), \forall x \in \Omega,
\end{array}\right.
$$

where $u_{0}: \Omega \rightarrow \mathbb{R}^{2}$ and $f: \Omega \times(0, T) \rightarrow \mathbb{R}^{2}$ are given data.

This problem is a vectorial one in analogy with the nonlinear system considered in Section 3.

2.1. An adaptive method in space and time. We aim to derive an adaptive code for the integration of (2.1). Our techniques will be inspired by the work of C. Johnson et al. (see [5, 6] in particular).

The spatial discretization is based on the space $S_{M}$ of trigonometric polynomials with values in $\mathbb{R}^{2}$ of degree $\leq M / 2$ in each variable. We denote by $P_{M}$ the $L^{2}(\Omega)$ projection onto $S_{M}$. We also set

$$
Q_{M}=I-P_{M} .
$$

The time discretization we use is a discontinuous Galerkin method of order zero (see Eriksson, Johnson and Thomée [8]). We let $N \in \mathbb{N}^{*}$ and introduce the following decomposition of $(0, T)$ :

$$
0=t_{0}<t_{1}<\cdots<t_{N}=T .
$$

We denote by $I_{n}=\left(t_{n-1}, t_{n}\right)_{n=1, \ldots, N}$ the $N$ subintervals of $(0, T)$, and set $k_{n}=$ $t_{n}-t_{n-1}$.

On each time interval $I_{n}$, the exact solution $u$ of problem (2.1) is approximated by $U_{n} \in S_{M_{n}}$. So, the approximate solution $U$ belongs to the following space $\mathcal{E}_{h}$ 


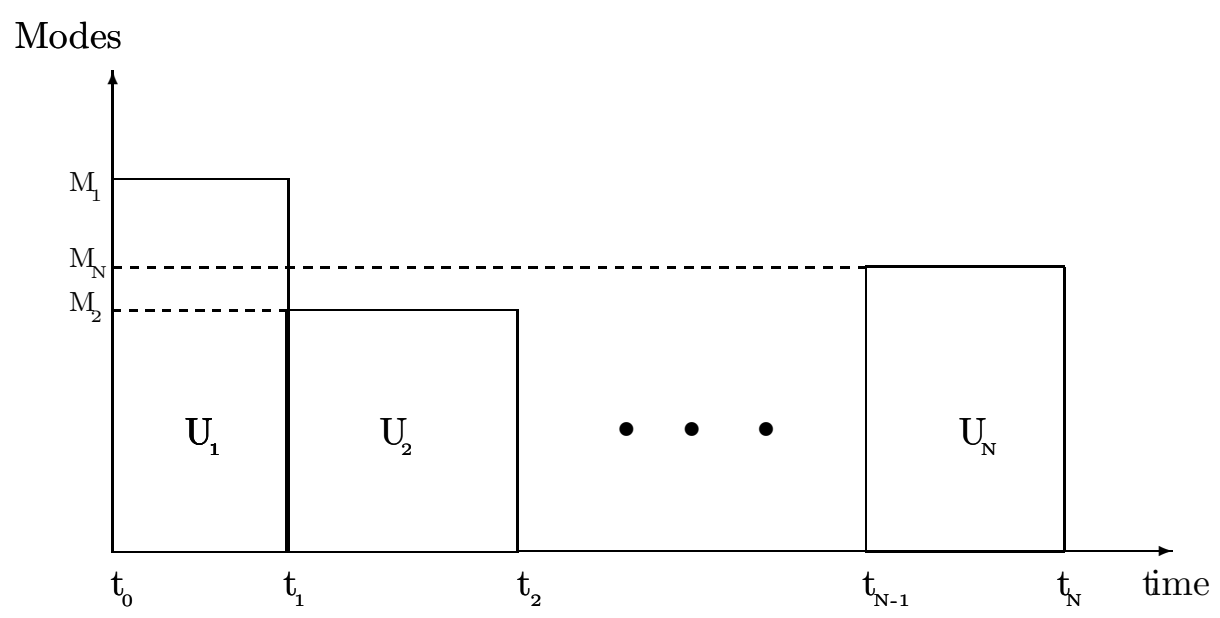

FIGURE 1. Description of approximation space $\mathcal{E}_{h}$

(see also Figure1):

$$
\mathcal{E}_{h}=\left\{U ; \forall n \in\{1, \ldots, N\},\left.U(t)\right|_{I_{n}}=U_{n} \in S_{M_{n}}\right\} .
$$

Then, the sequence $\left(U_{n}\right)_{n}$ is given by the following recursive formula:

$$
U_{n}-P_{M_{n}} U_{n-1}-k_{n} \Delta U_{n}=\int_{I_{n}} P_{M_{n}} f d t, n=1, \ldots, N,
$$

where

$$
U_{0}=u_{0}
$$

This scheme is a variant of the backward Euler scheme, where $f$ is integrated over time exactly.

We now aim to give an a posteriori error estimate for this scheme. We need some additional notation. We denote by $\mathbb{H}_{p}^{r}(\Omega)$ the space of functions which belong to $H_{\text {loc }}^{r}\left(\mathbb{R}^{2}\right)^{2}$ and are $\Omega$-periodic. The space $\mathbb{H}_{p}^{0}(\Omega)$ is equipped with the usual $L^{2}$-norm

$$
|u|=\left(\int_{\Omega} u(x) \cdot u(x) d x\right)^{1 / 2} .
$$

We also set

$$
\|u\|_{T}=\sup _{t \in(0, T)}|u(., t)| .
$$

The functions $k$ and $M$ are defined on $(0, \mathrm{~T})$ by:

$$
\left.k(t)\right|_{I_{n}}=k_{n},\left.M(t)\right|_{I_{n}}=M_{n}, \text { for } n=1, \ldots, N .
$$

Then we state the following 
Theorem 2.1. If $u_{0} \in \mathbb{H}_{p}^{1}(\Omega)$ and $f, f_{t}\left(=\frac{\partial f}{\partial t}\right) \in L^{\infty}\left(0, T ; \mathbb{H}_{p}^{0}(\Omega)\right)$, then the following error estimate holds:

$$
\begin{aligned}
& \max _{n=0, \ldots, N}\left|u\left(t_{n}\right)-U_{n}\right| \\
& \leq L_{N}\left\{\left\|k^{2} f_{t}\right\|_{T}+\max _{n=1, \ldots, N}\left|U_{n}-U_{n-1}\right|+4\left\|\frac{Q_{M} f}{M^{2}}\right\|_{T}+4 \max _{n=1, \ldots, N}\left|\frac{Q_{M_{n}} U_{n-1}}{k_{n} M_{n}^{2}}\right|\right\},
\end{aligned}
$$

where we set

$$
L_{N}=2+\frac{1}{2} \max _{n=1, \ldots, N} \sqrt{\log \left(\frac{t_{n}}{k_{n}}\right)}, U_{0}=u_{0} .
$$

We refer to Burie [1] for the proof of Theorem 2.1. See also the Appendix for the proof of analogous estimates for nonlinear equations.

The different terms arising in the a posteriori estimate (2.5) can be easily interpreted. Indeed the first two are due to the time discretization; in particular, since $\left|U_{n}-U_{n-1}\right| \simeq\left|k u_{t}\right|$, we see that the scheme is of order one in time. The next two terms are related to the spatial discretization. In particular, since $M_{n} \geq M_{n-1}$ implies $Q_{M_{n}} U_{n-1}=0$, the term $\frac{Q_{M_{n}} U_{n-1}}{k_{n} M_{n}^{2}}$ of (2.5) may be viewed as an estimate of the de-refining error.

Let TOL be a given tolerance. Using the above a posteriori estimate, we aim to devise an adaptive algorithm that computes an approximate solution $U$ of problem (2.1) satisfying

$$
\left|u\left(t_{n}\right)-U_{n}\right| \leq \mathrm{TOL}, \text { for } n=1, \ldots, N .
$$

Of course, the discretization parameters should be chosen so that the computational cost is optimal.

In view of (2.5), it is natural to introduce the following functions:

$$
\begin{aligned}
& \operatorname{Est}_{k_{n}}\left(k_{n}, M_{n}, U_{n}\right)=L_{n}\left\{k_{n}^{2}\left\|f_{t}\right\|_{I_{n}}+\left|U_{n}-U_{n-1}\right|\right\}, \\
& \text { Est }_{M_{n}}\left(k_{n}, M_{n}, U_{n}\right)=4 L_{n}\left\{M_{n}^{-2}\left\|Q_{M_{n}} f\right\|_{I_{n}}+\left|\frac{Q_{M_{n}} U_{n-1}}{k_{n} M_{n}^{2}}\right|\right\}, \\
& \text { Est }_{n}=\operatorname{Est}_{k_{n}}+\operatorname{Est}_{M_{n}}, \\
& \text { Est }_{T}=\max _{n=1, \ldots, N} \text { Est }_{n},
\end{aligned}
$$

where $n \in \mathbb{N}^{*}, L_{n}=2+1 / 2 \max _{i=1, \ldots, n} \sqrt{\log \left(t_{i} / k_{i}\right)}$, and $\|u\|_{I_{n}}=\sup _{t \in I_{n}}|u(., t)|$. Clearly, Est $T_{T}$ is equal to the right-hand side of the a posteriori estimate (2.5). Therefore, in order to guarantee the error control (2.6) it is sufficient to find the time steps $k_{n}$, the numbers of modes $M_{n}$ and the corresponding $U_{n}$ such that for each $n=1, \ldots, N$

$$
\operatorname{Est}_{n}\left(k_{n}, M_{n}, U_{n}\right) \leq \text { TOL. }
$$

At a typical time step, $k_{n}, M_{n}$ and $U_{n}$ are determined through an iterative procedure. Let us denote by $F_{n}$ the function giving $U_{n}$ in terms of $k_{n}, M_{n}$ and $U_{n-1}$ in (2.4); that is,

$$
U_{n}=F_{n}\left(k_{n}, M_{n}, U_{n-1}\right)=P_{M_{n}} U_{n-1}+k_{n} \Delta U_{n}+\int_{I_{n}} P_{M_{n}} f d t
$$


Then, note that $\operatorname{Est}_{k_{n}}$ (resp. Est $_{M_{n}}$ ) depends mainly on $k_{n}$ (resp. $M_{n}$ ). We introduce a sequence $\left(k_{n}^{j}, M_{n}^{j}, U_{n}^{j}\right)_{j \geq 0}$ that tends to $\left(k_{n}, M_{n}, U_{n}\right)$ satisfying

$$
\operatorname{Est}_{k_{n}}\left(k_{n}, M_{n}, U_{n}\right) \simeq \frac{9}{10} \mathrm{TOL}, \quad \operatorname{Est}_{M_{n}}\left(k_{n}, M_{n}, U_{n}\right) \simeq \frac{1}{10} \mathrm{TOL},
$$

as follows. Suppose that $\left(k_{n-1}, M_{n-1}, U_{n-1}\right)$ is known. We set

$$
\left(k_{n}^{0}, M_{n}^{0}\right)=\left(k_{n-1}, M_{n-1}\right) \quad \text { and } \quad U_{n}^{0}=F\left(k_{n}^{0}, M_{n}^{0}, U_{n-1}\right) .
$$

Next, for $j \geq 1, k_{n}^{j}$ is given by

$$
\left(k_{n}^{j}\right)^{2} L_{n}\left\{\left\|f_{t}\right\|_{I_{n}^{j-1}}+\frac{\left|U_{n}^{j-1}-U_{n-1}\right|}{\left(k_{n}^{j-1}\right)^{2}}\right\}=\frac{9}{10} \mathrm{TOL},
$$

where $I_{n}^{j-1}$ denotes the interval $\left(t_{n}, t_{n}+k_{n}^{j-1}\right)$. Next, $M_{n}^{j}$ is the smallest $M \in \mathbb{N}^{*}$ satisfying

$$
4 L_{n}\left\{M^{-2}\left\|Q_{M} f\right\|_{I_{n}^{j-1}}+\left|\frac{Q_{M} U_{n-1}}{k_{n}^{j} M^{2}}\right|\right\} \leq \frac{1}{10} \mathrm{TOL},
$$

and $U_{n}^{j}$ is given by

$$
U_{n}^{j}=F_{n}\left(k_{n}^{j}, M_{n}^{j}, U_{n-1}\right) .
$$

The procedure is reiterated until we reach the stopping condition

$$
\operatorname{Est}_{n}\left(k_{n}^{j}, M_{n}^{j}, U_{n}^{j}\right) \leq \text { TOL. }
$$

In this case, we then set $\left(k_{n}, M_{n}, U_{n}\right)=\left(k_{n}^{j}, M_{n}^{j}, U_{n}^{j}\right)$.

Thanks to the stopping condition (2.9d) and to (2.8), for each $n \in\{1, \ldots, N\}$, the error control (2.7) should be satisfied with near equality, which is necessary in view of the reliability and efficiency of the algorithm.

Moreover, if TOL is small enough the discretization parameters should slightly vary from one time step of the scheme to the next one. Therefore, the sequence $\left(k_{n}^{j}, M_{n}^{j}, U_{n}^{j}\right)_{j}$ should converge in very few iterations. We will check this property numerically.

Remark 2.2. It could seem more natural to ask for

$$
\operatorname{Est}_{k_{n}}\left(k_{n}, M_{n}, U_{n}\right) \simeq \mathrm{TOL} / 2, \quad \operatorname{Est}_{M_{n}}\left(k_{n}, M_{n}, U_{n}\right) \simeq \mathrm{TOL} / 2
$$

instead of (2.8). But, since the parameter $M_{n}$ is discrete, this choice would lead to $\operatorname{Est}_{M_{n}}\left(k_{n}, M_{n}, U_{n}\right) \ll \mathrm{TOL} / 2$; so the estimated error would often be about half the tolerance, which implies a loss of efficiency for the algorithm.

2.2. A multi-level adaptive method. The strategy of the multi-level method we will introduce consists in freezing the higher modes of the approximate solution during several iterations of the lower modes. As explained in the introduction, we expect that integrating the higher modes with a larger time-step will not spoil the overall accuracy of the method.

As in Section 2.1 we denote by $U$ the approximate solution of (2.1) and use the decomposition of $(0, T)$ in $N$ subintervals $I_{n}=\left(t_{n-1}, t_{n}\right)$ with $k_{n}=t_{n}-t_{n-1}$.

Now, concerning the spatial discretization, on each interval $I_{n}$ we are given two integers $m_{n}$ and $M_{n}$ such that $1<m_{n} \leq M_{n}$. The integer $m_{n}$ is called the cutoff mode. The approximate solution splits into a sum of two components, a low frequency one $V_{n}$ and a high frequency one $W_{n}$ :

$$
U_{n}=V_{n}+W_{n}, \quad V_{n} \in P_{m_{n}} S_{M_{n}} \text { and } W_{n} \in\left(P_{M_{n}}-P_{m_{n}}\right) S_{M_{n}}
$$




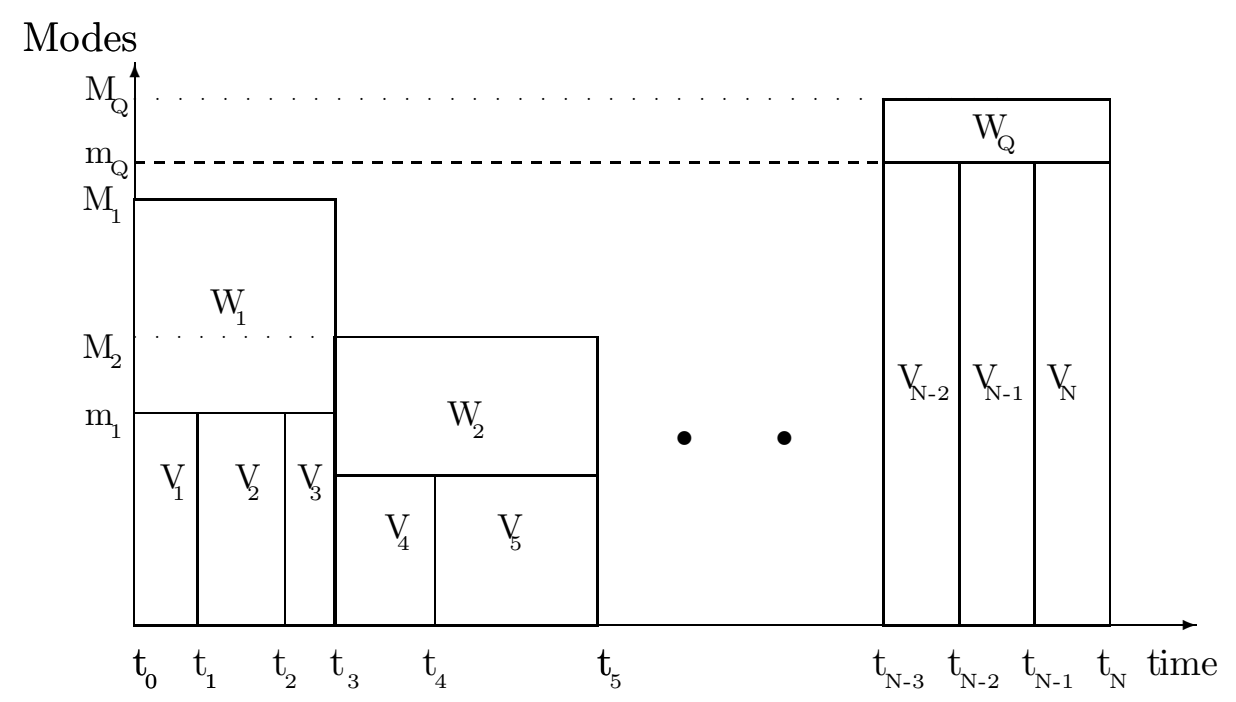

FIGURE 2. Description of approximation space $\mathcal{F}_{h}$

For each $q=1, \ldots, Q$, let $p_{q}$ be a nonnegative integer. The component $W_{n}$ is kept constant over $p_{q}$ successive intervals $I_{n}$. We denote by $J_{q}$ the union of these intervals, and by $K_{q}$ the length of $J_{q}$. Obviously, we have

$$
K_{q}=\sum_{n, I_{n} \subset J_{q}} k_{n}
$$

Since the parameters $m_{n}, M_{n}$ and $W_{n}$ are kept constant over $J_{q}$, they are denoted by $m_{q}, M_{q}$ and $W_{q}$.

The approximate solution $U$ of problem (2.1) lies in the space, (see also Figure 21),

$$
\begin{gathered}
\mathcal{F}_{h}=\left\{U ; \forall q \in\{1, \ldots, Q\}, \forall n \in\{1, \ldots, N\} \text { such that } I_{n} \subset J_{q},\right. \\
\left.\left.U\right|_{I_{n}}(t)=U_{n}=V_{n}+W_{q}, \text { where } V_{n} \in S_{m_{q}} \text { and } W_{q} \in\left(P_{M_{q}}-P_{m_{q}}\right) S_{M_{q}}\right\} .
\end{gathered}
$$

For $q \in\{1, \ldots, Q\}$, we denote by $n_{q}$ the index of the first interval $I_{n}$ contained in $J_{q}$; that is,

$$
\forall q=1, \ldots, Q, n_{q}=\min \left\{n \in\{1, \ldots, N\} \text { such that } I_{n} \subset J_{q}\right\} .
$$

The sequences $\left(V_{n}\right)_{n}$ and $\left(W_{q}\right)_{q}$ are given by the following recursive formulas:

$$
W_{q}-\left(P_{M_{q}}-P_{m_{q}}\right) U_{n_{q}-1}-K_{q} \Delta W_{q}=\int_{J_{q}}\left(P_{M_{q}}-P_{m_{q}}\right) f d t, \quad q=1, \ldots, Q,
$$

where

$$
U_{0}=u_{0}
$$


Note that the component $V_{n}$ is computed with the time-step $k_{n}$, whereas $W_{q}$ is computed with the time-step $K_{q}$. The equations (2.12 $\left.\mathrm{a}\right)$ and (2.12b) are coupled through the projection of $U_{n-1}$ on the new set of lower (resp. higher) modes.

We now give an a posteriori error estimate for this multilevel scheme. Let $k, K$, $m$ and $M$ be the functions defined on $(0, T)$ by

$$
\left.k(t)\right|_{I_{n}}=k_{n},\left.K(t)\right|_{J_{q}}=K_{q},\left.m(t)\right|_{J_{q}}=m_{q},\left.M(t)\right|_{J_{q}}=M_{q}
$$

The following theorem is proved in Burie [1]; see also the Appendix for nonlinear equations.

Theorem 2.3. If $u_{0} \in \mathbb{H}_{p}^{1}(\Omega)$ and $f, f_{t} \in L^{\infty}\left(0, T ; \mathbb{H}_{p}^{0}(\Omega)\right)$, then the following error estimate holds:

$$
\begin{aligned}
& \max _{n=0, \ldots, N}\left|u\left(t_{n}\right)-U_{n}\right| \\
& \leq L_{N}\left\{\left\|k^{2} f_{t}\right\|_{T}+\max _{n=1, \ldots, N}\left|U_{n}-U_{n-1}\right|+4\left\|\frac{Q_{M} f}{M^{2}}\right\|_{T}+4 \max _{q=1, \ldots, Q}\left|\frac{Q_{M_{q}} U_{n_{q}-1}}{k_{n_{q}} M_{q}^{2}}\right|\right. \\
& \left.\quad+\left\|\left(k^{2}+K^{2}\right)\left(P_{M}-P_{m}\right) f_{t}\right\|_{T}+2 \max _{q=1, \ldots, Q}\left|\left(P_{M_{q}}-P_{m_{q}}\right)\left(U_{n_{q}}-U_{n_{q}-1}\right)\right|\right\},
\end{aligned}
$$

where $n_{q}$ is given by (2.11) and

$$
L_{N}=2+\frac{1}{2} \max _{n=1, \ldots, N} \sqrt{\log \left(\frac{t_{n}}{k_{n}}\right)}, \quad U_{0}=u_{0} .
$$

It is interesting to compare estimate (2.13) with the corresponding one for the scheme (2.4). The first two lines in the right-hand side of (2.13) are similar to the estimate (2.5); in particular, we emphasize that the constants are the same ones. The other terms of (2.13) are clearly due to the multilevel strategy. They vanish if $m \equiv M$. Also, if $m$ is close to $M$, these terms are small in comparison with the previous ones. Indeed, in this case we have

$$
\left\|\left(k^{2}+K^{2}\right)\left(P_{M}-P_{m}\right) f_{t}\right\|_{T} \ll\left\|k^{2} f_{t}\right\|_{T} ;
$$

similarly, provided $k$ and $K$ are 'small enough',

$$
\begin{aligned}
2 \max _{q=1, \ldots, Q}\left|\left(P_{M_{q}}-P_{m_{q}}\right)\left(U_{n_{q}}-U_{n_{q}-1}\right)\right| & \simeq 2\left\|K\left(P_{M}-P_{m}\right) u_{t}\right\|_{T} \\
\ll \max _{n=1, \ldots, N}\left|\left(U_{n}-U_{n-1}\right)\right| & \simeq\left\|k u_{t}\right\|_{T} .
\end{aligned}
$$

We now aim to define an adaptive algorithm that yields an approximate solution $U$ of problem (2.1) such that

$$
\left|u\left(t_{n}\right)-U_{n}\right| \leq \mathrm{TOL}, \text { for } n=1, \ldots, N
$$


where TOL is some given tolerance. Recalling (2.13), for $n \in\{1, \ldots, N\}$ and $q \in\{1, \ldots, Q\}$ we introduce the following functions:

$$
\begin{aligned}
& \text { Est }_{k_{n}}=L_{n}\left\{k_{n}^{2}\left\|f_{t}\right\|_{I_{n}}+\left|U_{n}-U_{n-1}\right|\right\}, \\
& \text { Est }_{M_{q}}=4 L_{n}\left\{M_{q}^{-2}\left\|Q_{M_{q}} f\right\|_{J_{q}}+\left|\frac{Q_{M_{q}} U_{n_{q}-1}}{k_{n_{q}} M_{q}^{2}}\right|\right\}, \\
& \text { Est }_{M L_{q}}=2\left\|K_{q}^{2}\left(P_{M_{q}}-P_{m_{q}}\right) f_{t}\right\|_{J_{q}}+2\left|\left(P_{M_{q}}-P_{m_{q}}\right)\left(U_{n_{q}}-U_{n_{q}-1}\right)\right|, \\
& \text { Est }_{q}=\max _{n \text { such that } I_{n} \subset J_{q}} \text { Est }_{k_{n}}+\operatorname{Est}_{M_{q}}+\operatorname{Est}_{M L_{q}}, \\
& \text { Est }_{T}=\max _{q=1, \ldots, Q} \text { Est }_{q},
\end{aligned}
$$

where $n_{q}$ is given by (2.11), $U_{n}=V_{n}+W_{q}$ and $L_{n}=2+1 / 2 \max _{i=1, \ldots, n} \sqrt{\log \left(t_{i} / k_{i}\right)}$ (the dependence of $L_{n}$ upon $J_{q}$ is neglected).

Thus defined, Est ${ }_{T}$ is greater than or equal to the right-hand side of (2.13). So, in order to guarantee the error control (2.14), it is sufficient to find for each $q \in\{1, \ldots, Q\}$

$$
K_{q}, M_{q}, m_{q} \text {, and } k_{n} \text { for all } n \text { such that } I_{n} \subset J_{q},
$$

and the corresponding approximations $W_{q}$ and $\left(V_{n}\right)_{n, I_{n} \subset J_{q}}$ such that

$$
\operatorname{Est}_{q}\left(k_{n}, K_{q}, M_{q}, m_{q}, V_{n}+W_{q}\right) \leq \mathrm{TOL} .
$$

At a typical time step $q$, by analogy with the one-level method, we use an iterative procedure that converges to parameters and corresponding approximate solutions satisfying

$$
\begin{gathered}
\operatorname{Est}_{k_{n}}\left(k_{n}, K_{q}, \ldots\right) \leq \mathrm{TOL}_{1}, \quad \operatorname{Est}_{M_{q}}\left(k_{n}, K_{q}, \ldots\right) \leq \mathrm{TOL}_{2}, \\
\operatorname{Est}_{M L_{q}}\left(k_{n}, K_{q}, \ldots\right) \leq \mathrm{TOL}_{3},
\end{gathered}
$$

with $\mathrm{TOL}_{1}+\mathrm{TOL}_{2}+\mathrm{TOL}_{3}=\mathrm{TOL}$.

Here, Est $k_{n}$ and Est $_{M_{q}}$ will allow us to define the time step $k_{n}$ (for the lower modes) and the number of modes $M_{n}$. Then the other term Est $_{M L_{q}}$ should allow us to find the time step $K_{q}$ (for the higher modes) and the cut-off mode $m_{q}$. However, this quantity does not seem to be sufficient to yield both parameters (especially if $f_{t} \equiv 0$ ). Therefore one needs to introduce a supplementary condition. Here we choose to fix the number of iterations of $V$ during which $W$ is frozen. That is, the ratio $K_{q} / k_{n}$ is kept constant; we set

$$
\frac{K_{q}}{k_{n}}=p \in \mathbb{N}^{*},
$$

where the value of $p$ is chosen a priori. In practice, during the iterative procedure, once the parameter $k_{n}^{j}$ is given by $\operatorname{Est}_{k_{n}}\left(k_{n}^{j}\right) \simeq \mathrm{TOL}_{1}$, we set $K_{q}^{j}=p k_{n}^{j}$, and $m_{q}^{j}$ is given by condition $\operatorname{Est}_{M L_{q}}\left(K_{q}^{j}, m_{q}^{j}\right) \simeq \mathrm{TOL}_{3}$.

This procedure is rather natural, since $\operatorname{Est}_{M L_{q}}\left(K_{q}, m_{q}\right) \leq \mathrm{TOL}_{3}$ always has the trivial solution $m_{q}=M_{q}$. Clearly, if the algorithm gives $m_{q}<M_{q}$, this will show that the higher modes of $U$ can indeed be integrated with a larger time step without any loss in accuracy.

Remark 2.4. The above method involves two levels, but the principle can easily be extended to several levels. Indeed, let $N$ be some number of levels and let $m_{i}$ be 
$N$ integers such that $1<m_{1}<\cdots<m_{N}$. The approximate solution can be split into the sum

$$
U=V+W_{2}+\cdots+W_{N},
$$

where $V \in S_{m_{1}}$ and $W_{i} \in\left(P_{m_{i}}-P_{m_{i-1}}\right) S_{m_{N}}$. The component $V$ is then integrated with a time step $k$, and each $W_{i}$ with a time step $p_{i} k$, where the $p_{i}$ are integers satisfying $1<p_{2}<\cdots<p_{N}$. The $p_{i}$ are chosen a priori; for instance a natural choice is $p_{i}=2^{i-1}$. Then, the adaptive algorithm gives the values of the different cut-off modes $m_{i}$.

We now introduce the algorithm. Let $\varepsilon>0$ be a small parameter. We set

$$
\mathrm{TOL}_{1}=(1-\varepsilon) \frac{9 \mathrm{TOL}}{10} ; \quad \mathrm{TOL}_{2}=(1-\varepsilon) \frac{\mathrm{TOL}}{10} ; \quad \mathrm{TOL}_{3}=\varepsilon \mathrm{TOL} .
$$

This choice will provide values of $k$ and $M$ close to those in the one-level method, so that it will be possible to compare the numerical performances of the two schemes. For the numerical tests, we set $\varepsilon=1 / 25$.

The algorithm is composed of two steps. At a typical time step $q$, we recall that $n_{q}$ is defined by

$$
n_{q}=\min \left\{n \in\{1, \ldots, N\} \text { such that } I_{n} \subset J_{q}\right\} .
$$

Suppose that $\left(k_{n_{q}-1}, K_{q-1}, M_{q-1}, m_{q-1}\right)$ and $U_{n_{q}-1}=V_{n_{q}-1}+W_{q-1}$ are known. First, we compute parameters $k_{n_{q}}, K_{q}, M_{q}, m_{q}$ and the corresponding components $V_{n_{q}}$ and $W_{q}$ of $U_{n_{q}}$ given by (2.12a) and (2.12b). Next, for all $n$ such that $I_{n} \subset J_{q}$, since $K_{q}, M_{q}, m_{q}$ and $W_{q}$ are known and fixed, we only have to compute parameters $k_{n}$ and the corresponding component $V_{n}$ of $U_{n}=V_{n}+W_{q}$ given by (2.12a).

More precisely, we first introduce a sequence

$$
\left(k_{n_{q}}^{j}, K_{q}^{j}, M_{q}^{j}, m_{q}^{j}, U_{n_{q}}^{j}=V_{n_{q}}^{j}+W_{q}^{j}\right)_{j \geq 0},
$$

where $V_{n_{q}}^{j}$ and $W_{q}^{j}$ are computed in terms of $k_{n_{q}}^{j}, K_{q}^{j}, M_{q}^{j}, m_{q}^{j}$ and $U_{n_{q}-1}$ thanks to $2.12 \mathrm{a})$ and $2.12 \mathrm{~b})$. It is defined as follows. We set $\left(k_{n_{q}}^{0}, K_{q}^{0}, M_{q}^{0}, m_{q}^{0}\right)=$ $\left(k_{n_{q}-1}, K_{q-1}, M_{q-1}, m_{q-1}\right)$ and compute the corresponding $U_{n_{q}}^{0}=V_{n_{q}}^{0}+W_{q}^{0}$. Next, for $j \geq 1, k_{n_{q}}^{j}$ is given by

$$
\left(k_{n_{q}}^{j}\right)^{2} L_{n_{q}}\left\{\left\|f_{t}\right\|_{I_{n_{q}}^{j-1}}+\frac{\left|U_{n_{q}}^{j-1}-U_{n_{q}-1}\right|}{\left(k_{n_{q}}^{j-1}\right)^{2}}\right\}=\mathrm{TOL}_{1},
$$

where $I_{n_{q}-1}^{j-1}$ denotes the interval $\left(t_{n}, t_{n}+k_{n_{q}}^{j-1}\right)$. Then,

$$
K_{q}^{j}=p k_{n_{q}}^{j},
$$

and $M_{q}^{j}$ is the smallest $M \in \mathbb{N}^{*}$ satisfying

$$
4 L_{n_{q}}\left\{M^{-2}\left\|Q_{M} f\right\|_{J_{q}^{j-1}}+\left|\frac{Q_{M} U_{n_{q}-1}}{k_{n_{q}}^{j-1} M^{2}}\right|\right\} \leq \mathrm{TOL}_{2} .
$$

Finally, $m_{q}^{j}$ is the smallest $m \leq M_{q}^{j}$ satisfying

$$
2 L_{n_{q}}\left\{\left(K_{q}^{j}\right)^{2}\left\|\left(P_{M_{q}^{j}}-P_{m}\right) f_{t}\right\|_{J_{q}^{j-1}}+\left|\left(P_{M_{q}^{j-1}}-P_{m}\right)\left(U_{n_{q}}^{j-1}-U_{n_{q}-1}\right)\right|\right\} \leq \mathrm{TOL}_{3},
$$

and we compute the corresponding $U_{n_{q}}^{j}=V_{n_{q}}^{j}+W_{q}^{j}$ given by (2.12 $\mathrm{a}$ ) and (2.12b). 
This first procedure is reiterated until we reach the stopping condition

$$
\left(\operatorname{Est}_{k_{n_{q}}}+\operatorname{Est}_{M_{q}}+\operatorname{Est}_{M L_{q}}\right)\left(k_{n_{q}}^{j}, K_{q}^{j}, M_{q}^{j}, m_{q}^{j}, U_{n_{q}}^{j}\right) \leq \text { TOL. }
$$

In this case, we set $\left(k_{n_{q}}, K_{q}, M_{q}, m_{q}, U_{n_{q}}\right)=\left(k_{n_{q}}^{j}, K_{q}^{j}, M_{q}^{j}, m_{q}^{j}, U_{n_{q}}^{j}\right)$.

Next, we perform the remaining iterations of $V_{n}$. For this, we introduce a new variable $\operatorname{Sum} k$, and set $\operatorname{Sum} k=k_{n_{q}}$. As long as Sumk $<K_{q}$, for each $n$, the parameter $k_{n-1}$ and $U_{n-1}=V_{n-1}+W_{q}$ being known, we determine $k_{n}$ and $V_{n}$ as follows. We set $k_{n}^{0}=k_{n-1}$ and compute the corresponding component $V_{n}^{0}$ thanks to (2.12a). Next, for $j \geq 1, k_{n}^{j}$ is given by

$$
\left(k_{n}^{j}\right)^{2} L_{n}\left\{\left\|f_{t}\right\|_{I_{n}^{j-1}}+\frac{\left|U_{n}^{j-1}-U_{n-1}\right|}{\left(k_{n}^{j-1}\right)^{2}}\right\}=\mathrm{TOL}_{1},
$$

and we compute $U_{n}^{j}=V_{n}^{j}+W_{q}$, where $V_{n}^{j}$ is given by $(2.12$ ).

This procedure is reiterated until the stopping condition

$$
\operatorname{Est}_{k_{n}}\left(k_{n}^{j}, U_{n}^{j}\right) \leq \mathrm{TOL}_{1}
$$

is satisfied. In this case, we set $\left(k_{n}, U_{n}\right)=\left(k_{n}^{j}, U_{n}^{j}\right)$ and $\operatorname{Sum} k=\operatorname{Sum} k+k_{n}$, and perform the next iteration of $V_{n}$.

2.3. Numerical tests. We first give some indications on the practical implementation of the one-level adaptive algorithm, which easily extend to the multilevel algorithm. To allow Fast Fourier Transform (FFT) algorithms, the levels of modes $M$ belong to

$$
G_{M}=\left\{M \in \mathbb{N} ; M=2^{a} 3^{b} 5^{c}, a, b, c \in \mathbb{N} \text { with } a \geq 2 \text { and } 4 \leq M \leq M_{\max }\right\}
$$

where $M_{\max }=128$. Then, assuming $M \ll M_{\max }$, we approximate $u_{0}$ by $P_{M_{\max }} u_{0}$.

According to the scheme (2.4), at each iteration $n$, we need to compute the quantity $P_{M_{n}} U_{n-1}$, where $U_{n-1} \in S_{M_{n-1}}$. If $M_{n}>M_{n-1}, P_{M_{n}} U_{n-1}$ is obtained by padding $U_{n-1}$ with zeroes. On the contrary, if $M_{n}<M_{n-1}, P_{M_{n}} U_{n-1}$ is simply obtained by truncation. Next, the quantities $\left|U_{n}-U_{n-1}\right|$ and $\left|Q_{M_{n}} U_{n-1}\right|$ for the a

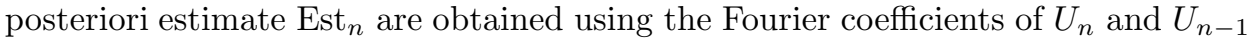
and the Parseval equality.

The first solution of (2.1) we consider is defined by $f \equiv 0$ and

$$
\left(u_{0}\right)_{1}\left(x_{1}, x_{2}\right)=\left(u_{0}\right)_{2}\left(x_{1}, x_{2}\right)=5 \exp \left(-5\left(\left(x_{1}-\pi\right)^{2}+\left(x_{2}-\pi\right)^{2}\right)\right) .
$$

The initial condition $u_{0}$ is a smooth 'approximation' of a $\delta$-function at $\left(x_{1}, x_{2}\right)=$ $(\pi, \pi)$ (see Figure 3).

We choose $T=1.5$ and $\mathrm{TOL}=0.001$. The results are given in Figure 5 for the one-level method, and in Figure 6 for the multilevel one, where $p=3$. In Figure 5 are displayed versus time:

- upper-left quadrant: time-steps $k$,

- upper-right quadrant: number of modes $M$, and $j_{\max }$ (points), the maximum number of iterations needed by the algorithm to find the discretization parameters,

- lower-left quadrant: $L^{2}$-norms of real (continuous line) and estimated error (points),

- lower-right quadrant: $L^{2}$-norm of the solution $u$ of (2.1). 
Figure [6 is similar to Figure 5] except for the upper-right quadrant, where we display the total number of modes $M$, and the cut-off mode $m$ (dashed line).

As expected, we first notice that the discretization parameters $k$ and $M$ given by both algorithms are roughly equal. Therefore, further displayed tests will only concern the multilevel method.

Next, in Figure 5 we find that $j_{\max }$ is equal to one most often, i.e. the search for discretization parameters loop converges in one single iteration. This allows us to minimize the cost of the adaptive algorithm.

Defining the efficiency index by

$$
\mathrm{Eff}=\frac{\|u-U\|_{T}}{\operatorname{Est}_{T}}
$$

for all performed tests, we found that $E f f \simeq 0.2$. For solution 1, we computed it for several values of TOL, and, checked that it does not depend on TOL:

\begin{tabular}{|c||c|c|c|c|c|c|}
\hline TOL & $10^{-1}$ & $5.10^{-2}$ & $10^{-2}$ & $5.10^{-3}$ & $10^{-3}$ & $5.10^{-4}$ \\
\hline \hline Eff & 0.20 & 0.19 & 0.22 & 0.22 & 0.23 & 0.21 \\
\hline
\end{tabular}

Concerning computing time, the following table compares both methods for various tolerances and values of $p$. All computations were performed on a HP 9000/715 work station.

\begin{tabular}{|c|l||c||c|c|c|c|c|}
\hline \multicolumn{2}{|c||}{ Solution $1(\mathrm{~T}=1.5)$} & 1 level & $p=3$ & $p=5$ & $p=7$ & $p=9$ & $p=10$ \\
\hline \hline \multirow{2}{*}{$\begin{array}{c}\text { TOL } \\
=10^{-3}\end{array}$} & CPU time (seconds) & $78 \mathrm{~s}$ & $65 \mathrm{~s}$ & $63 \mathrm{~s}$ & $64 \mathrm{~s}$ & - & - \\
\cline { 2 - 8 } & M-L time / 1-L time & 1 & 0.83 & 0.81 & 0.82 & & \\
\hline \hline $\begin{array}{c}\text { TOL } \\
=5.10^{-4}\end{array}$ & CPU time (seconds) & $189 \mathrm{~s}$ & $139 \mathrm{~s}$ & $140 \mathrm{~s}$ & $135 \mathrm{~s}$ & $134 \mathrm{~s}$ & $136 \mathrm{~s}$ \\
\cline { 2 - 8 } & M-L time / 1-L & 1 & 0.74 & 0.74 & 0.71 & 0.71 & 0.72 \\
\hline
\end{tabular}

In this case, the gain in computing time allowed by the multilevel strategy reaches $30 \%$. We notice that the smaller TOL is, the better the gain is. Actually, this fact is general, as we will show later.

The second solution we consider is defined by (see Figure 4)

$$
\begin{aligned}
& \left(u_{0}\right)_{1}\left(x_{1}, x_{2}\right)=\left(u_{0}\right)_{2}\left(x_{1}, x_{2}\right)=\left(-8 \pi^{4} / 15+4 \pi^{2} x_{2}^{2}-4 \pi x_{2}^{3}+x_{2}^{4}\right) / 100, \\
& f_{1}\left(x_{1}, x_{2}\right)=f_{2}\left(x_{1}, x_{2}\right)=\exp \left(-0.1\left(\left|x_{1}-\pi\right|^{2}+\left|x_{2}-\pi\right|^{2}\right)\right)(\cos (\pi t / 2))^{5} .
\end{aligned}
$$

We choose $T=8$ and $\mathrm{TOL}=0.01$. The result of the multilevel method is shown in Figure 7

With $T=120$ (i.e. 30 periods of $f$ ), we check the stability of the multilevel method for TOL $=0.05$ (see Figure 8).

Concerning computing time, we have the following table.

\begin{tabular}{|c|l||c||c|c|c|c|}
\hline \multicolumn{2}{|c|}{ Solution $2(\mathrm{~T}=8)$} & 1 level & $p=3$ & $p=5$ & $p=7$ & $p=9$ \\
\hline \hline \multirow{2}{*}{$\begin{array}{c}\text { TOL } \\
=10^{-2}\end{array}$} & CPU time (seconds) & $29 \mathrm{~s}$ & $18.5 \mathrm{~s}$ & $17 \mathrm{~s}$ & $17 \mathrm{~s}$ & - \\
\cline { 2 - 7 } & M-L time/ 1-L time & 1 & 0.64 & 0.59 & 0.59 & - \\
\hline \hline \multirow{2}{*}{$\begin{array}{c}\text { TOL } \\
=10^{-3}\end{array}$} & CPU time (seconds) & $1250 \mathrm{~s}$ & $685 \mathrm{~s}$ & $576 \mathrm{~s}$ & $498 \mathrm{~s}$ & $520 \mathrm{~s}$ \\
\cline { 2 - 7 } & M-L time/ 1-L time & 1 & 0.55 & 0.46 & 0.40 & 0.42 \\
\hline
\end{tabular}


For this solution, the gain in computing time reaches $60 \%$, and, again increases when TOL decreases.

Indeed, as shown on Figure 9, $p$ being a constant, as TOL decreases the total number of modes $M$ increases since a finer refinement is needed, while the cut-off value $m$ given by the algorithm does not change. More precisely, there exists a function $m c(t)$, which does not depend on TOL, such that if $M(t) \geq m c(t)$ then $m(t) \simeq m c(t)$.

Therefore, compared with the one-level method, the smaller TOL is, the larger the number of modes integrated with a time-step $p k>k$ is, and finally, the greater the gain is.

We heuristically justify this behaviour. At a typical time-step $n$, let us first assume that

$$
\left|k_{n}^{2} f_{t}\left(t_{n}\right)\right| \gg\left|U_{n}-U_{n-1}\right|
$$

and, also,

$$
\left|p^{2} k_{n}^{2}\left(P_{M}-P_{m}\right) f_{t}\left(t_{n}\right)\right| \gg\left|\left(P_{M}-P_{m}\right)\left(U_{n_{q}}-U_{n_{q}-1}\right)\right| .
$$

Then, due to the a posteriori estimate (2.13) and to the construction of the multilevel algorithm, we have

$$
\left|k_{n}^{2} f_{t}\left(t_{n}\right)\right| \simeq \frac{9 \mathrm{TOL}}{10}, \quad 2 p^{2}\left|k_{n}^{2}\left(P_{M}-P_{m}\right) f_{t}\left(t_{n}\right)\right| \simeq \frac{\mathrm{TOL}}{25},
$$

and so, dividing the former term by the latter one, we see that

$$
p^{2} \frac{\left|\left(P_{M}-P_{m}\right) f_{t}\left(t_{n}\right)\right|}{\left|f_{t}\left(t_{n}\right)\right|} \simeq \frac{1}{45}
$$

Next, assume that

$$
\left|k_{n}^{2} f_{t}\left(t_{n}\right)\right| \ll\left|U_{n}-U_{n-1}\right|
$$

and

$$
\left|U_{n}-U_{n-1}\right| \simeq k_{n}\left|u_{t}\left(t_{n}\right)\right|
$$

and also

$$
\left|\left(P_{M}-P_{m}\right)\left(U_{n_{q}}-U_{n_{q}-1}\right)\right| \simeq\left|\left(P_{M}-P_{m}\right)\left(W_{q}-W_{q-1}\right)\right| \simeq p k_{n}\left|u_{t}\left(t_{n}\right)\right| .
$$

As before, we then obtain

$$
p \frac{\left|\left(P_{M}-P_{m}\right) u_{t}\left(t_{n}\right)\right|}{\left|u_{t}\left(t_{n}\right)\right|} \simeq \frac{1}{45} .
$$

So, in both cases, we have found an 'equation' that does not depend on TOL, linking $p$ and $m$. These two equations explain the existence of $m c(t)$. (Of course, this behaviour is strongly related to the fact that, for the multilevel algorithm, $p$ is a constant given by the user.)

In conclusion, we have checked that the multilevel method allows a significant gain in computing time, compared with an optimal adaptive one-level method. Moreover, we have shown that the smaller the required accuracy is, the greater the gain is. 


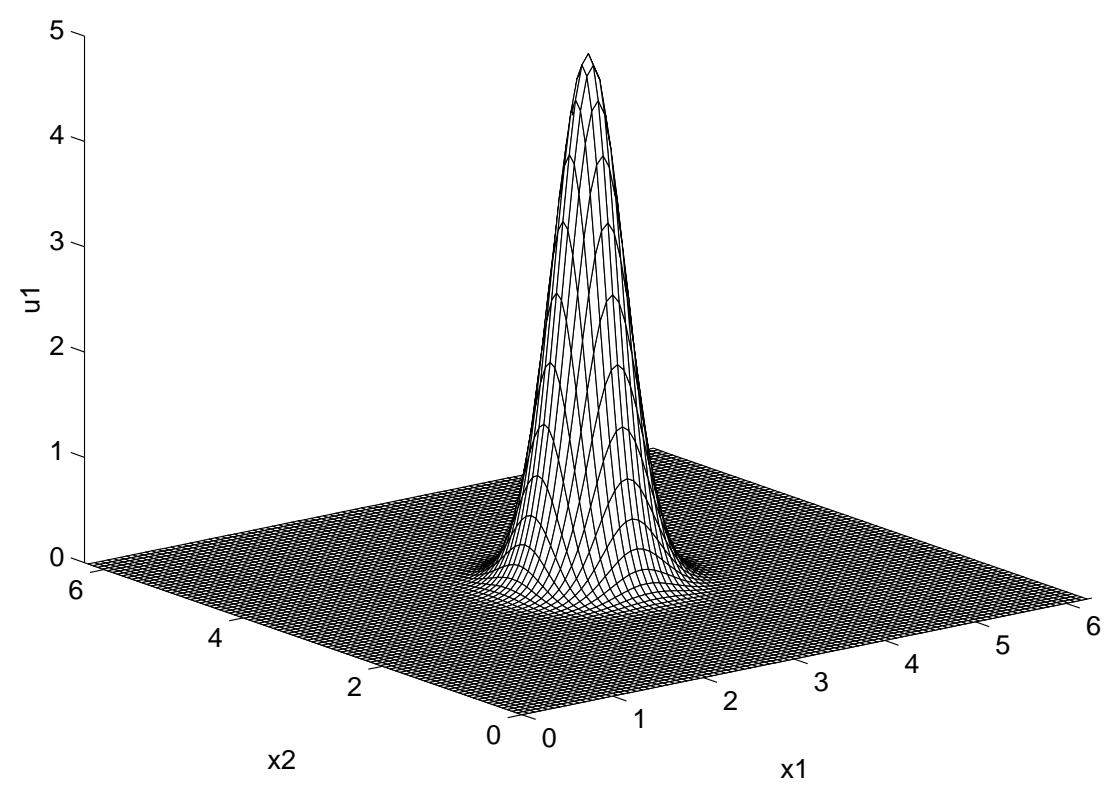

FiguRE 3 . First component of the initial condition $u_{0}$ of solution 1

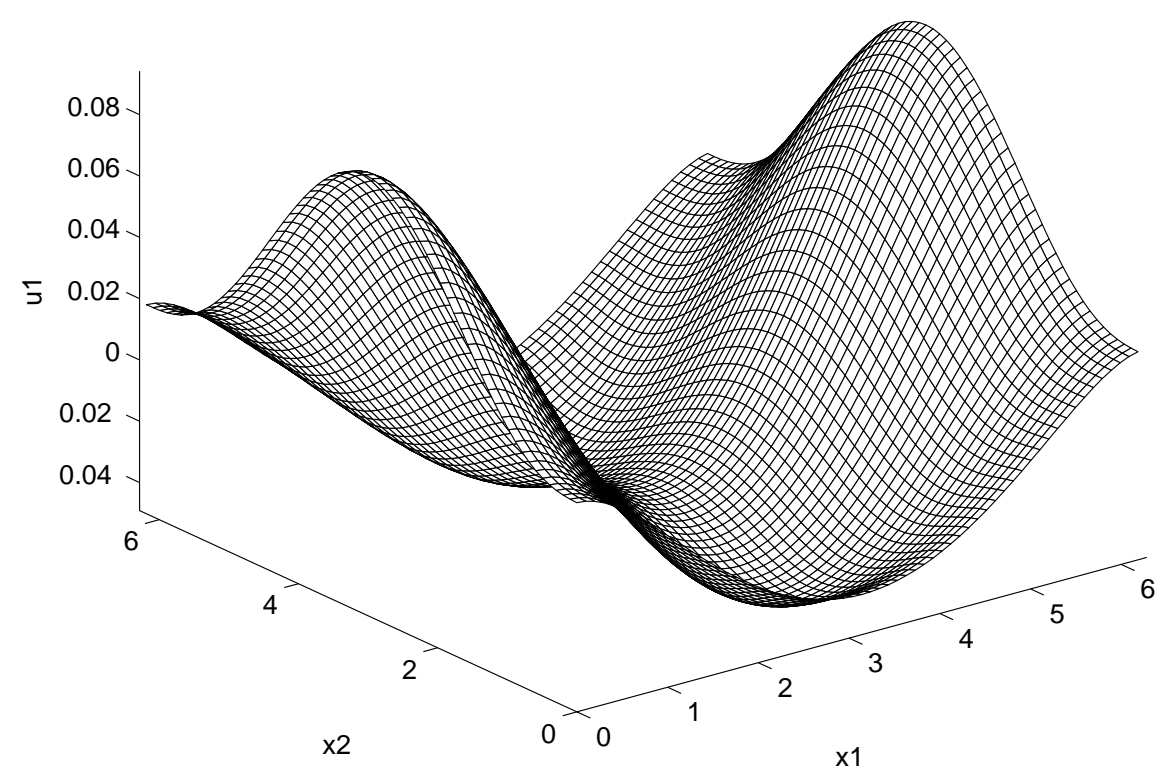

FIGURE 4. First component of solution 2 at $T=2$ 

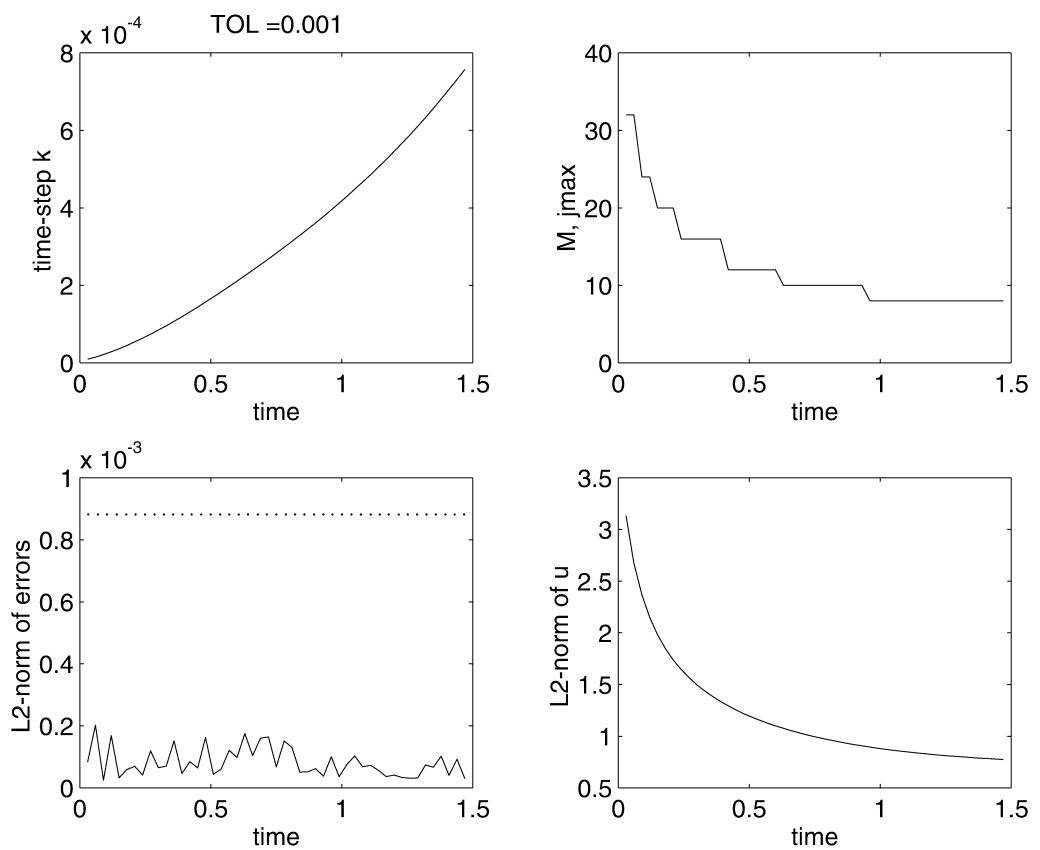

Figure 5. Solution 1 , TOL $=0.001, T=1.5$
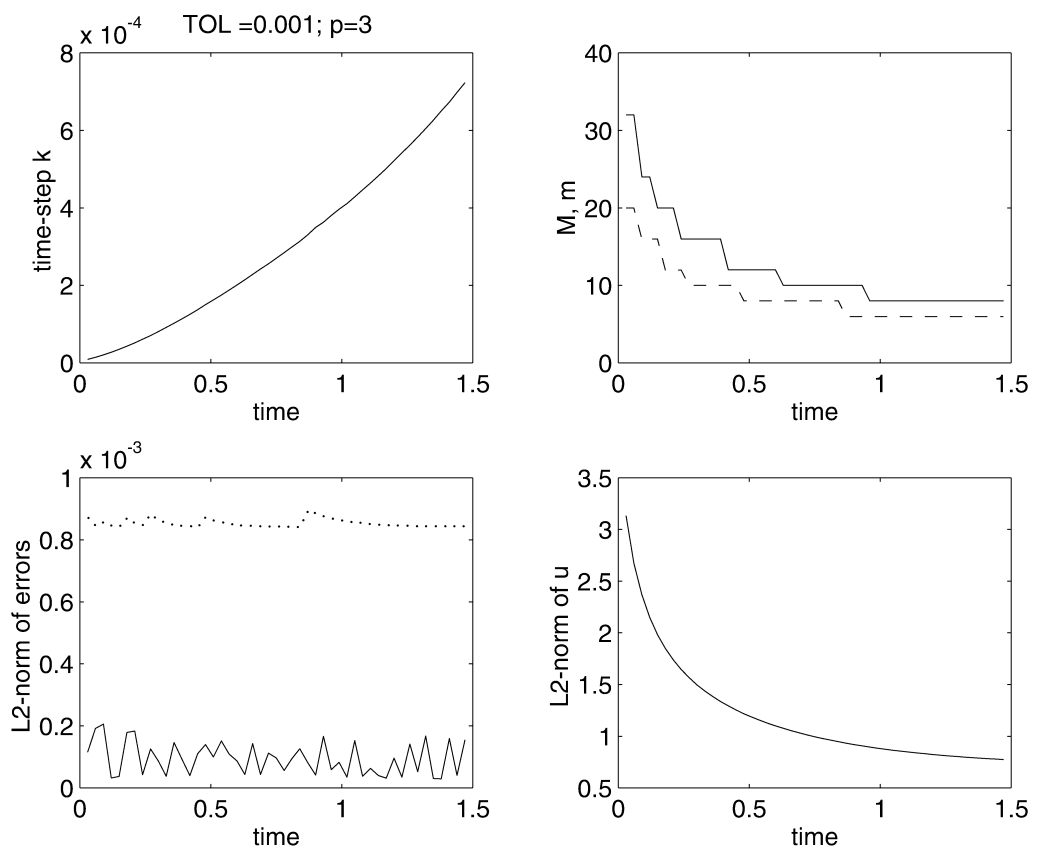

Figure 6 . Solution 1 , TOL $=0.001, p=3, T=1.5$ 

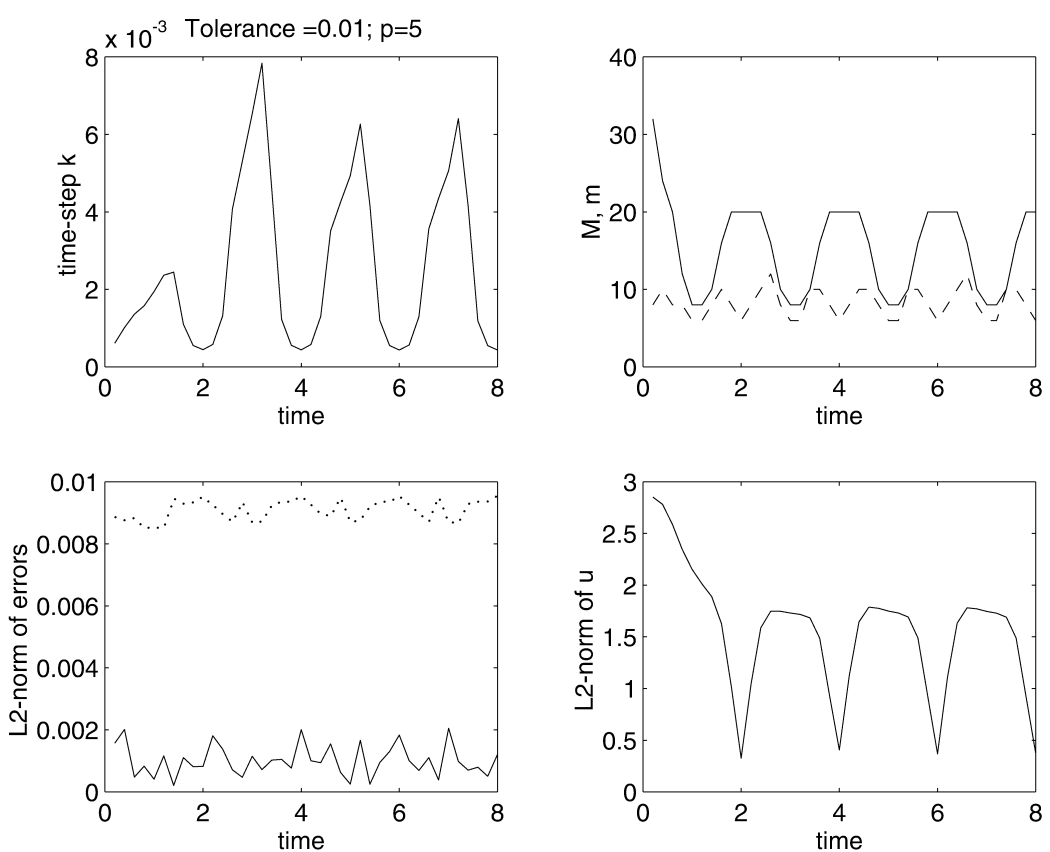

Figure 7. Solution 2, TOL $=0.01, p=5, T=8$
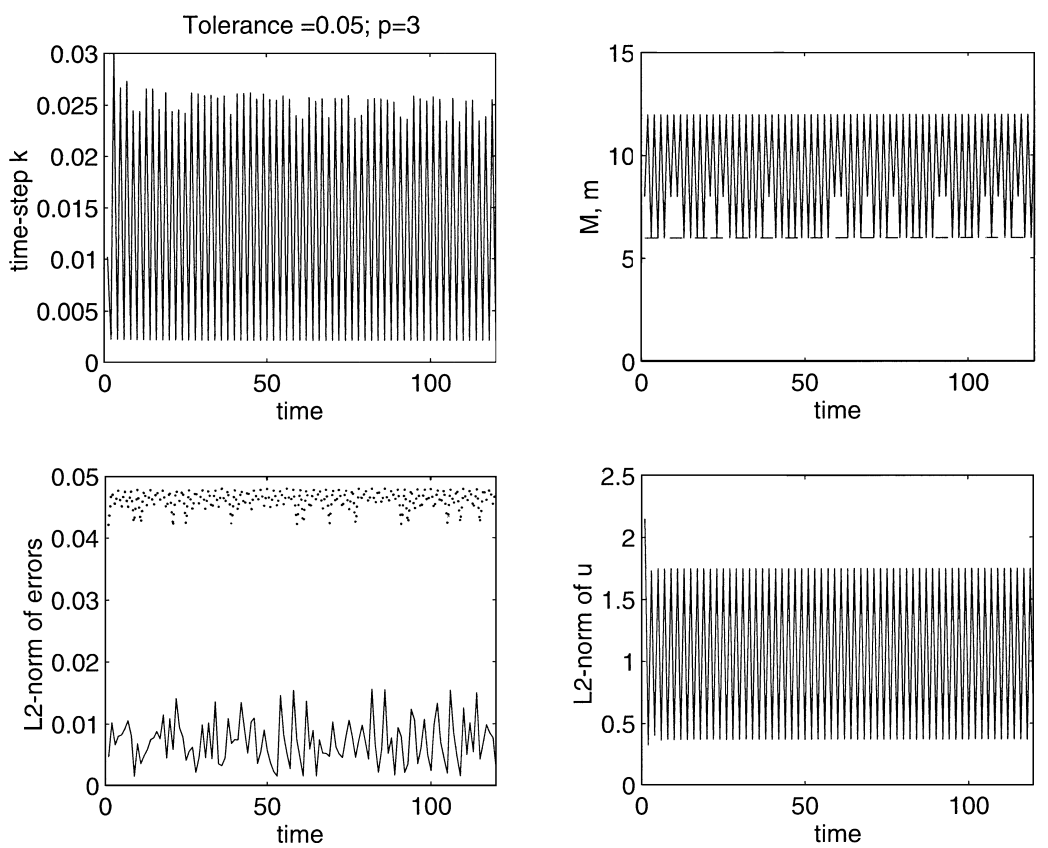

Figure 8. Solution 2, stability test 

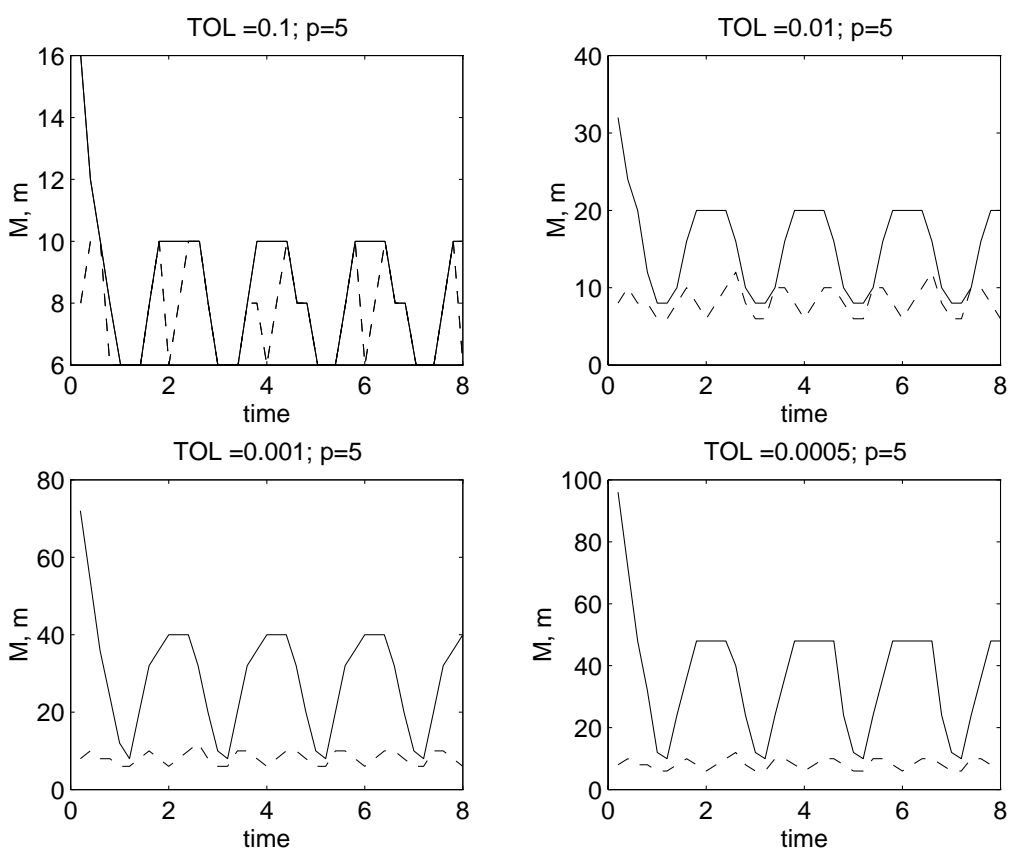

Figure 9. Solution $2, p=5, m(t)$ does not depend on TOL

\section{A NONLINEAR PROBLEM}

Let $\Omega=(0,2 \pi)^{2}$ and $T>0$. In this part, we consider the following nonlinear problem.

Find $\left(u=\left(\begin{array}{l}u_{1} \\ u_{2}\end{array}\right), p\right)$ such that

$$
\begin{cases}\frac{\partial u_{1}}{\partial t}-\Delta u_{1}+u_{1} \frac{\partial u_{1}}{\partial x}+\frac{\partial p}{\partial x}=f_{1}, & \text { in } \Omega \times(0, T), \\ \frac{\partial u_{2}}{\partial t}-\Delta u_{2}+\frac{\partial p}{\partial y}=f_{2}, & \text { in } \Omega \times(0, T), \\ \operatorname{div} u=0 \text { and } \int_{\Omega} u(x, t) d x=0, & \text { in } \Omega \times(0, T), \\ u(., t) \text { and } p(., t) \text { are } \Omega \text {-periodic, } & t \in(0, T), \\ \left.u\right|_{t=0}=u_{0} . & \end{cases}
$$

To simplify the notation, hereafter the following abstract formulation of (3.1) in the space of divergence-free functions will be considered:

$$
\left\{\begin{array}{l}
u_{t}+A u+B(u)=f, \\
u(0)=u_{0},
\end{array}\right.
$$

where $A$ denotes the Stokes operator and $B(u)$ the projection of the nonlinearity on the space of divergence-free functions. Note that problem (3.1) satisfies the 
classical property of the Navier-Stokes equations

$$
\int_{\Omega} B(u) \cdot u d x=0
$$

Hereafter, we will need the following functional spaces:

$$
\begin{aligned}
& H=\left\{u \in \mathbb{H}_{p}^{0}(\Omega), \int_{\Omega} u(x) d x=0 \text { and } \operatorname{div} u=0\right\}, \\
& V=\left\{u \in \mathbb{H}_{p}^{1}(\Omega), \int_{\Omega} u(x) d x=0 \text { and } \operatorname{div} u=0\right\} .
\end{aligned}
$$

We also recall that $D(A)=H \cap \mathbb{H}_{p}^{2}(\Omega)$. In the sequel, we assume that

$$
u_{0} \in D(A) \text { and } f, f_{t} \in L^{\infty}(0, T ; H),
$$

which is sufficient to ensure that the solution $u$ of (3.1) belongs to

$\mathcal{E}=\left\{u: \Omega \times[0, T] \rightarrow \mathbb{R}^{2}, u \in \mathcal{C}([0, T] ; V) \cap L^{2}(0, T ; D(A))\right.$ and $\left.u_{t} \in L^{2}(0, T ; H)\right\}$.

3.1. An adaptive method in space and time. We use notation analogous to that of Section 2.1 for the heat equation. In particular, $S_{M_{n}}$ still denotes the space of trigonometric polynomials with values in $\mathbb{R}^{2}$ of degree $\leq M_{n} / 2$ in each variable, but supplemented with a divergence-free condition. Also, $P_{M_{n}}$ denotes the projection on this space. Taking this difference into account, the approximate solution $U$ lies in space $\mathcal{E}_{h}$ defined by (2.3). The sequence $\left(U_{n}\right)_{n}$ is advanced in time thanks to the following scheme:

$$
U_{n}-P_{M_{n}} U_{n-1}+k_{n} A U_{n}+k_{n} P_{M_{n}} B\left(U_{n-1}\right)=\int_{I_{n}} P_{M_{n}} f d t, n=1, \ldots, N
$$

with

$$
U_{0}=u_{0}
$$

This scheme is a variant of the discontinuous Galerkin method, in which the nonlinear term is computed explicitly, for implementation convenience.

The techniques for the a posteriori analysis of problem (3.1) are inspired by the work of C. Johnson et al. (see [7, 10] in particular). For a general nonlinear problem, this analysis requires the introduction of stability constants, whose computation is a nontrivial difficulty. Indeed, they are defined through the solution of an associated linear problem that depends on the solution $u$ and its approximation $U$. Usually, this dual problem has to be solved numerically.

However in the case of problem (3.1), these stability constants can be estimated from above with realistic and explicit bounds. This would not be true for the Navier-Stokes equations, for which the corresponding bounds involve terms like $\exp \left(c T\|\nabla u\|_{\infty}\right)$, where $\|\cdot\|_{\infty}$ denotes the $L^{\infty}\left(0, T ; L^{\infty}(\Omega)\right)$-norm. Such a bound cannot be used numerically, since this would lead to over-refined discretizations.

Here, the stability constants are defined through the solution $(\varphi, q)$ of the following associated problem. 
Let $\hat{T}<T$. Find $\left(\varphi=\left(\begin{array}{l}\varphi_{1} \\ \varphi_{2}\end{array}\right), q\right)$ such that

$$
\begin{cases}-\varphi_{t}-\Delta \varphi+B^{*}(u, U ; \varphi)+\nabla q=0, & \text { in } \Omega \times(0, \hat{T}), \\ \operatorname{div} \varphi=0 \text { and } \int_{\Omega} \varphi(x, t) d x=0, & \text { in } \Omega \times(0, \hat{T}), \\ \varphi(., t) \text { and } q(., t) \text { are } \Omega \text {-periodic, } & t \in(0, \hat{T}), \\ \left.\varphi\right|_{t=\hat{T}}=\varphi_{\hat{T}}, & \end{cases}
$$

where we set

$$
B^{*}(u, U ; \varphi)=\left(\begin{array}{c}
\frac{\partial\left(U_{1}-u_{1}\right)}{\partial x} \varphi_{1}+u_{1} \frac{\partial \varphi_{2}}{\partial y} \\
0
\end{array}\right) .
$$

Using standard Galerkin techniques, one can check that, if $\varphi_{\hat{T}} \in V$, then $\varphi \in$ $\mathcal{E} \cap \mathcal{C}([0, \hat{T}) ; D(A))$.

Through the solution $\varphi$ of (3.6), we are now able to define the following stability constants $C_{i}^{S}, i=1, \ldots, 5$.

$$
C_{1}^{S}(T, u, U)=\sup _{0 \leq \hat{T} \leq T} \sup _{\varphi_{\hat{T}} \in H} \frac{\max \left(\|\varphi\|_{(0, \hat{T})}, \sup _{0 \leq \varepsilon \leq 1 / e}|\log \varepsilon|^{-1 / 2} \int_{0}^{\hat{T}-\varepsilon}\left|\varphi_{t}\right| d t\right)}{\left|\varphi_{\hat{T}}\right|}
$$

$$
\begin{gathered}
C_{2}^{S}(T, u, U)=\sup _{0 \leq \hat{T} \leq T} \sup _{\varphi_{\hat{T}} \in H} \frac{\sup _{0 \leq \varepsilon \leq 1 / e}|\log \varepsilon|^{-1 / 2} \int_{0}^{\hat{T}-\varepsilon}|\Delta \varphi| d t}{\left|\varphi_{\hat{T}}\right|} \\
C_{3}^{S}(T, u, U)=\sup _{0 \leq \hat{T} \leq T} \sup _{\varphi_{\hat{T}} \in H} \frac{\|\left.\int_{t}^{\hat{T}} \Delta \varphi(s) d s\right|_{(0, \hat{T})}}{\left|\varphi_{\hat{T}}\right|} \\
C_{4}^{S}(T, u, U)=\sup _{1 \leq \hat{N} \leq N} \sup _{\varphi_{\hat{T}} \in H} \frac{\left|\log k_{\hat{N}}\right|^{-1 / 2} \sum_{n=1}^{\hat{N}} k_{n}\left|\Delta \varphi\left(t_{n-1}\right)\right|}{\left|\varphi_{\hat{T}}\right|} \\
C_{5}^{S}(T, u, U)=\sup _{0 \leq \hat{T} \leq T} \sup _{\hat{T} \in H} \frac{\int_{0}^{\hat{T}}|\varphi(s)| d s}{\left|\varphi_{\hat{T}}\right|}
\end{gathered}
$$

where |.| denotes the $\left(L^{2}(\Omega)\right)^{2}$ norm and

$$
\|u\|_{I}=\sup _{t \in I}|u(., t)| .
$$

If the discretization is fine enough, we may assume that the constants $C_{i}^{S}(T, u, U)$ can be approximated by $C_{i}^{S}(T, u, u)$. Then, as already mentioned, these constants can be bounded from above as follows (proof omitted):

$$
\begin{aligned}
& C_{1}^{S}(T, u, u) \leq \max \left\{2+\min (1, \sqrt{2 T})\left\|u_{1}\right\|_{\infty}, 3 / 2+2 \sqrt{2}\left\|u_{1}\right\|_{\infty}\right\} \\
& C_{2}^{S}(T, u, u) \leq 3 / 2+2 \sqrt{2}\left\|u_{1}\right\|_{\infty} \\
& C_{3}^{S}(T, u, u) \leq 3+2 \min (1, \sqrt{2 T})\left\|u_{1}\right\|_{\infty}, \\
& C_{5}^{S}(T, u, u) \leq\left\{2+\min (1, \sqrt{2 T})\left\|u_{1}\right\|_{\infty}\right\} \min (1, T),
\end{aligned}
$$


where $\left\|u_{1}\right\|_{\infty}$ denotes the $L^{\infty}\left(0, T ; L^{\infty}(\Omega)\right)$-norm of $u_{1}$. Moreover, since the constant $C_{4}^{S}$ is the analogue of $C_{2}^{S}$ with the integral replaced by a rectangle formula, in the implementation we also use the bound (3.9) for $C_{4}^{S}(T, u, u)$

We can now state the following a posteriori error estimate.

Theorem 3.1. If $u_{0} \in D(A)$, and if $f, f_{t} \in L^{\infty}(0, T ; H)$, then the following error estimate holds:

$$
\begin{aligned}
\mid u\left(t_{N}\right)- & U_{N} \mid \leq\left(2+L_{N}\right) C_{1}^{S}(T, u, U)\left\{\left\|k^{2} f_{t}\right\|_{T}+\max _{n \in\{1, \ldots, N\}}\left|U_{n}-U_{n-1}\right|\right\} \\
& +4 L_{N} C_{2}^{S}(T, u, U)\left\|\frac{Q_{M} f}{M^{2}}\right\|_{T}+4 L_{N} C_{4}^{S}(T, u, U) \max _{n \in\{1, \ldots, N\}}\left|\frac{Q_{M_{n}} U_{n-1}}{k_{n} M_{n}^{2}}\right| \\
& +4 L_{N} C_{2}^{S}(T, u, U) \max _{n \in\{1, \ldots, N\}}\left|\frac{Q_{M_{n}} B\left(U_{n}\right)}{M_{n}^{2}}\right| \\
& +C_{5}^{S}(T, u, U) \max _{n \in\{1, \ldots, N\}}\left|P_{M_{n}}\left(B\left(U_{n}\right)-B\left(U_{n-1}\right)\right)\right| \\
& +4 C_{3}^{S}(T, u, U)\left\{\left\|\frac{Q_{M} f}{M^{2}}\right\|_{I_{N}}+\left\|\frac{k Q_{M} f_{t}}{M^{2}}\right\|_{I_{N}}+\left|\frac{Q_{M_{N}} B\left(U_{N}\right)}{M_{N}^{2}}\right|\right\},
\end{aligned}
$$

where we have set

$$
L_{N}=\max _{n \in\{1, \ldots, N\}} \sqrt{\left|\log k_{n}\right|}, \quad U_{0}=u_{0}
$$

The constants $C_{i}^{S}(T, u, U)$ are defined by (3.8).

The proof of this theorem is given in the Appendix.

We now interpret the different terms arising in the right-hand side of (3.10). Up to the stability constants, the first two lines of (3.10) are similar to the estimate (2.5) of the linear problem.

The third and fourth lines of (3.10) are composed of terms involving the nonlinearity $B$. The first (resp. second) one is due to the spatial (resp. time) discretization. Again, since

$$
\left|P_{M_{n}}\left(B\left(U_{n}\right)-B\left(U_{n-1}\right)\right)\right| \simeq k_{n}\left|\frac{\partial B(u)}{\partial t}\left(t_{n}\right)\right|,
$$

we see that the scheme is of order one in time.

Finally, the last line is composed of $L^{\infty}$ norms on the interval $I_{N}$. Apart from this, the corresponding terms are analogous to previous terms of (3.10).

We can now introduce an adaptive algorithm for approximating problem (3.1) with some given tolerance. Of course, we want the numerical cost to be optimal.

The algorithm is built by analogy with the one devised for the linear problem. For a nonnegative $n$, we define the following functions:

$$
\begin{aligned}
\operatorname{Est}_{k_{n}}\left(k_{n}, M_{n}, U_{n}\right)= & \left(2+L_{n}\right) C_{1}^{S}\left(k_{n}^{2}\left\|f_{t}\right\|_{I_{n}}+\left|U_{n}-U_{n-1}\right|\right) \\
& +C_{5}^{S}\left|P_{M_{n}}\left(B\left(U_{n}\right)-B\left(U_{n-1}\right)\right)\right|, \\
\operatorname{Est}_{M_{n}}\left(k_{n}, M_{n}, U_{n}\right)= & 4\left(L_{n} C_{2}^{S}+C_{3}^{S}\right) M_{n}^{-2}\left(\left\|Q_{M_{n}} f\right\|_{I_{n}}+\left|Q_{M_{n}} B\left(U_{n}\right)\right|\right) \\
& +4 L_{n} C_{4}^{S}\left|\frac{Q_{M_{n}} U_{n-1}}{k_{n} M_{n}^{2}}\right|+4 C_{3}^{S} k_{n} M_{n}^{-2}\left\|Q_{M_{n}} f_{t}\right\|_{I_{n}},
\end{aligned}
$$




$$
\begin{aligned}
& \text { Est }_{n}=\text { Est }_{k_{n}}+\text { Est }_{M_{n}}, \\
& \text { Est }_{T}=\max _{n \in\{1, \ldots, N\}} \text { Est }_{n},
\end{aligned}
$$

where $L_{n}=\max _{i \in\{1, \ldots, n\}} \sqrt{\left|\log k_{i}\right|}$. The constants $C_{i}^{S}$ are defined by (3.8) with $(T, u, U)=\left(t_{n}, u, U\right)$.

Then, at a typical time step, suppose that $\left(k_{n-1}, M_{n-1}, U_{n-1}\right)$ is known. To find $k_{n}, M_{n}$, and the corresponding $U_{n}$, we introduce a sequence $\left(k_{n}^{j}, M_{n}^{j}, U_{n}^{j}\right)_{j>0}$, where $U_{n}^{j}$ is computed in terms of $k_{n}^{j}, M_{n}^{j}$ and $U_{n-1}$, thanks to the scheme (3.5), as follows. First, we set $\left(k_{n}^{0}, M_{n}^{0}\right)=\left(k_{n-1}, M_{n-1}\right)$, and compute $U_{n}^{0}$. Next, for $j \geq 1$, $k_{n}^{j}$ is given by

$$
\begin{aligned}
\left(k_{n}^{j}\right)^{2}\{ & \left(2+L_{n}\right) C_{1}^{S}\left(\left\|f_{t}\right\|_{I_{n}^{j-1}}+\frac{\left|U_{n}^{j-1}-U_{n-1}\right|}{\left(k_{n}^{j-1}\right)^{2}}\right) \\
& \left.+C_{5}^{S} \frac{\left|P_{M_{n}^{j-1}}\left(B\left(U_{n}^{j-1}\right)-B\left(U_{n-1}\right)\right)\right|}{\left(k_{n}^{j-1}\right)^{2}}\right\}=\frac{9}{10} \mathrm{TOL},
\end{aligned}
$$

where $I_{n}^{j-1}$ denotes the interval $\left(t_{n}, t_{n}+k_{n}^{j-1}\right)$. Next, $M_{n}^{j}$ is the smallest $M \in \mathbb{N}^{*}$ satisfying

$$
\begin{aligned}
& 4\left(L_{n} C_{2}^{S}+C_{3}^{S}\right) M^{-2}\left\{\left\|Q_{M} f\right\|_{I_{n}^{j-1}}+\left|Q_{M_{n}^{j-1}} B\left(U_{n}^{j-1}\right)\right|\right\} \\
+ & 4 L_{n} C_{4}^{S}\left|\frac{Q_{M} U_{n-1}}{k_{n}^{j} M^{2}}\right|+4 C_{3}^{S} k_{n}^{j} M^{-2}\left\|Q_{M} f_{t}\right\|_{I_{n}^{j-1}} \leq \frac{1}{10} \mathrm{TOL},
\end{aligned}
$$

and we compute $U_{n}^{j}$ by (3.5).

This procedure is reiterated until we reach the stopping condition

$$
\operatorname{Est}_{n}\left(k_{n}^{j}, M_{n}^{j}, U_{n}^{j}\right) \leq \text { TOL. }
$$

In this case, we set $\left(k_{n}, M_{n}, U_{n}\right)=\left(k_{n}^{j}, M_{n}^{j}, U_{n}^{j}\right)$.

3.2. A multilevel adaptive method. We keep the notation of Section 2.2 for the heat equation, except that the polynomials of $S_{M_{n}}$ now satisfy a divergence free condition as in Section 3.3 So, the approximate solution $U$ of (3.1) belongs to the space $\mathcal{F}_{h}$ defined by (2.10).

For $q \in\{1, \ldots, Q\}$, we recall that $n_{q}$ is the index of the first interval $I_{n}$ contained in $J_{q}$, that is,

$$
\forall q \in \mathbb{N}_{q}, n_{q}=\min \left\{n \in\{1, \ldots, N\} \text { such that } I_{n} \subset J_{q}\right\} .
$$

Then, the sequences $\left(V_{n}\right)_{n}$ and $\left(W_{q}\right)_{q}$ are recursively defined by

$$
V_{n}-P_{m_{n}} U_{n-1}+k_{n} A V_{n}+k_{n} P_{m_{n}} B\left(U_{n-1}\right)=\int_{I_{n}} P_{m_{n}} f(s) d s, n=1, \ldots, N,
$$

$$
\begin{aligned}
& W_{q}-\left(P_{M_{q}}-P_{m_{q}}\right) U_{n_{q}-1}+K_{q} A W_{q} \\
& \quad+K_{q}\left(P_{M_{q}}-P_{m_{q}}\right) B\left(U_{n_{q}-1}\right)=\int_{J_{q}}\left(P_{M_{q}}-P_{m_{q}}\right) f(s) d s, q=1, \ldots, Q,
\end{aligned}
$$

with

$$
U_{0}=u_{0}
$$


Equations (3.12 $\mathrm{a})$ and $(3.12 \mathrm{~b})$ are coupled, on one hand, through the projection on the new set of lower (resp. higher) modes of $U_{n-1}$, and, on the other hand, through the nonlinear term $B$. For both $V_{n}$ and $W_{q}$, the nonlinear term is computed explicitly.

The following theorem gives an a posteriori estimate for this scheme; we refer the reader to the Appendix for its proof.

Theorem 3.2. Let $u_{0} \in D(A)$ and $f \in L^{2}(0, T ; H)$. If $f, f_{t} \in L^{\infty}(0, T ; H)$, then the following error estimate holds:

$$
\begin{aligned}
\mid u\left(t_{N}\right)- & U_{N} \mid \leq\left(2+L_{N}\right) C_{1}^{S}(T, u, U)\left\{\left\|k^{2} f_{t}\right\|_{T}+\max _{n \in\{1, \ldots, N\}}\left|U_{n}-U_{n-1}\right|\right\} \\
& +4 L_{N} C_{2}^{S}(T, u, U)\left\|\frac{Q_{M} f}{M^{2}}\right\|_{T}+4 L_{N} C_{4}^{S}(T, u, U) \max _{q \in\{1, \ldots, Q\}}\left|\frac{Q_{M_{q}} U_{n_{q}-1}}{k_{n_{q}} M_{q}^{2}}\right| \\
& +4 L_{N} C_{2}^{S}(T, u, U) \max _{n \in\{1, \ldots, N\}}\left|\frac{Q_{M_{n}} B\left(U_{n}\right)}{M_{n}^{2}}\right| \\
& +C_{5}^{S}(T, u, U) \max _{n \in\{1, \ldots, N\}}\left|P_{m_{n}}\left(B\left(U_{n}\right)-B\left(U_{n-1}\right)\right)\right| \\
& +4 C_{3}^{S}(T, u, U)\left\{\left\|\frac{Q_{M} f}{M^{2}}\right\|_{I_{N}}+\left\|\frac{k Q_{M} f_{t}}{M^{2}}\right\|_{I_{N}}+\left|\frac{Q_{M_{N}} B\left(U_{N}\right)}{M_{N}^{2}}\right|\right\} \\
& +\left(2+L_{N}\right) C_{1}^{S}(T, u, U)\left\|K^{2}\left(P_{M}-P_{m}\right) f_{t}\right\|_{T} \\
& +\left(2+L_{N}\right) C_{1}^{S}(T, u, U) \max _{q \in\{1, \ldots, Q\}}\left|\left(P_{M_{q}}-P_{m_{q}}\right)\left(U_{n_{q}}-U_{n_{q}-1}\right)\right| \\
& +C_{5}^{S}(T, u, U) \max _{q}\left\{\max _{n \text { s. } t . I_{n} \subset J_{q}}\left|\left(P_{M_{q}}-P_{m_{q}}\right)\left(B\left(U_{n}\right)-B\left(U_{n_{q}-1}\right)\right)\right|\right\}
\end{aligned}
$$

where

$$
L_{N}=\max _{n \in\{1, \ldots, N\}} \sqrt{\left|\log k_{n}\right|}, \quad U_{0}=u_{0} .
$$

The constants $C_{i}^{S}(T, u, U)$ are defined by (3.8).

Let us compare estimate (3.13) with the one for the scheme (3.5). The first five lines of the right-hand side of (3.13) are analogous to the estimate (3.10).

The other terms of (3.13) are due to the multilevel strategy. Up to the stability constants, the first two terms are similar to the two last terms of (2.13). As previously noted, if $m$ is close to $M$, they are small in comparison with previous corresponding terms of (3.13). This result is still valid for the last term of (3.13) since, if $m \simeq M$, then

$$
\begin{aligned}
\max _{q \in\{1, \ldots, Q\}}\left|\left(P_{M_{q}}-P_{m_{q}}\right)\left(B\left(U_{n_{q}-1}\right)-B\left(U_{n}\right)\right)\right| & \simeq\left\|K\left(P_{M}-P_{m}\right) B(u)_{t}\right\|_{T} \\
& \ll \max _{n \in\{1, \ldots, N\}}\left|B\left(U_{n}\right)-B\left(U_{n-1}\right)\right| \simeq\left\|k B(u)_{t}\right\|_{T} .
\end{aligned}
$$

An adaptive algorithm can be built by using (3.13). It has some common features with the one for the heat equation, that is, (2.15). In particular, the number $p$ of iterations of $V$ during which $W$ is frozen is again fixed and chosen a priori. For the sake of brevity, we do not present this algorithm here and refer to Burie [1]. 
3.3. Numerical tests. We again give some indications on the implementation of the algorithms. Compared with the linear case, the new ingredients are the stability constants (3.8), and, the quadratic nonlinear term $B$, which is computed thanks to a pseudo-spectral method.

For both methods, in the estimators the stability constants $C_{i}^{S}\left(t_{n}, u, U\right)$ are replaced by their upper bounds (3.9).

The Fourier coefficient of $\varphi$ associated to the mode $l=\left(l_{1}, l_{2}\right) \in \mathbb{Z}^{2}$ is denoted by $\mathcal{F}_{l}(\varphi)$. We also define

$$
|l|=\sqrt{l_{1}^{2}+l_{2}^{2}}, \quad|l|_{\infty}=\max \left(\left|l_{1}\right|,\left|l_{2}\right|\right) .
$$

The projection of $\varphi$ on the divergence-free and zero-mean functions is easily expressed by

$$
P_{\operatorname{div}}(\varphi)(x)=\sum_{l \in \mathbb{Z}^{2}, l \neq 0}\left(\mathcal{F}_{l}(\varphi)-\frac{l}{|l|^{2}}\left(l \cdot \mathcal{F}_{l}(\varphi)\right)\right) e^{i l \cdot x} .
$$

One-level method. Since $U_{n-1} \in S_{M_{n-1}}$ (= vector of trigonometric polynomials of degree $\left.\leq M_{n-1} / 2\right), B\left(U_{n-1}\right)$ belongs to $S_{2 M_{n-1}}$. To compute all the Fourier coefficients of $B\left(U_{n-1}\right)$, before using the pseudo-spectral method, it would be necessary to pad the Fourier coefficients of $U_{n-1}$ for modes $l$ such that $M_{n-1} / 2<|l|_{\infty} \leq M_{n-1}$ with zeroes.

Actually, we use the $3 / 2$ rule (see for instance Canuto, Hussaini, Quarteroni, and Zang [3]). This method is a standard one in order to remove the aliasing error due to the pseudo-spectral method. It provides the Fourier coefficients of $B\left(U_{n-1}\right)$ for $|l|_{\infty} \leq 3 M_{n-1} / 4$, and for $|l|_{\infty} \leq M_{n-1} / 2$ the aliasing error is removed. Of course, to allow a FFT algorithm, the quantities $3 M / 2$ belong to $G_{M}$ defined by (2.16).

Then, assuming that $M_{n} \leq 3 M_{n-1} / 2$ (the variation of $M$ between two time steps is small), the computation of $U_{n}$ through formula (3.5) is achievable.

Concerning now the computation of the a posteriori estimate, the nonlinear terms are computed by using their Fourier coefficients thanks to the Parseval equality. In particular, we write

$$
\begin{aligned}
\left|\left(I-P_{M_{n}}\right) B\left(U_{n}\right)\right| & \simeq\left|\left(P_{3 M_{n} / 2}-P_{M_{n}}\right) B\left(U_{n}\right)\right| \\
& =2 \pi \sqrt{\sum_{M_{n} / 2<|l|_{\infty} \leq 3 M_{n} / 4}\left|\mathcal{F}_{l}\left(B\left(U_{n}\right)\right)\right|^{2}} .
\end{aligned}
$$

Multi-level method. At a typical time step $n$, to find $V_{n}$ and evaluate the a posteriori estimator, we need to compute $P_{m_{n}} B\left(U_{n-1}\right)$. We assume that $3 m_{n} \geq$ $M_{n-1}$. Although $U_{n-1}$ belongs to $S_{M_{n-1}}$, we compute $P_{m_{n}} B\left(U_{n-1}\right)$ with FFT based on $\left(3 m_{n} / 2\right)^{2}$ points rather than $\left(3 M_{n-1} / 2\right)^{2}$, which is compulsory if we wish to allow the multi-level method to be faster than the one-level.

For this, we proceed as follows. First, by analogy with the $3 / 2$ rule used for the one-level method, we start with $P_{3 m_{n} / 2} U_{n-1}$. Then, we project the Fourier coefficients for modes between $3 m_{n} / 2$ and $3 m_{n}$ upon the modes lower than $3 m_{n} / 2$ (see Burie and Marion [2]). So, if $3 m_{n} \geq M_{n-1}$, the nonlinear term $P_{m_{n}} B\left(U_{n-1}\right)$ is exactly computed. 


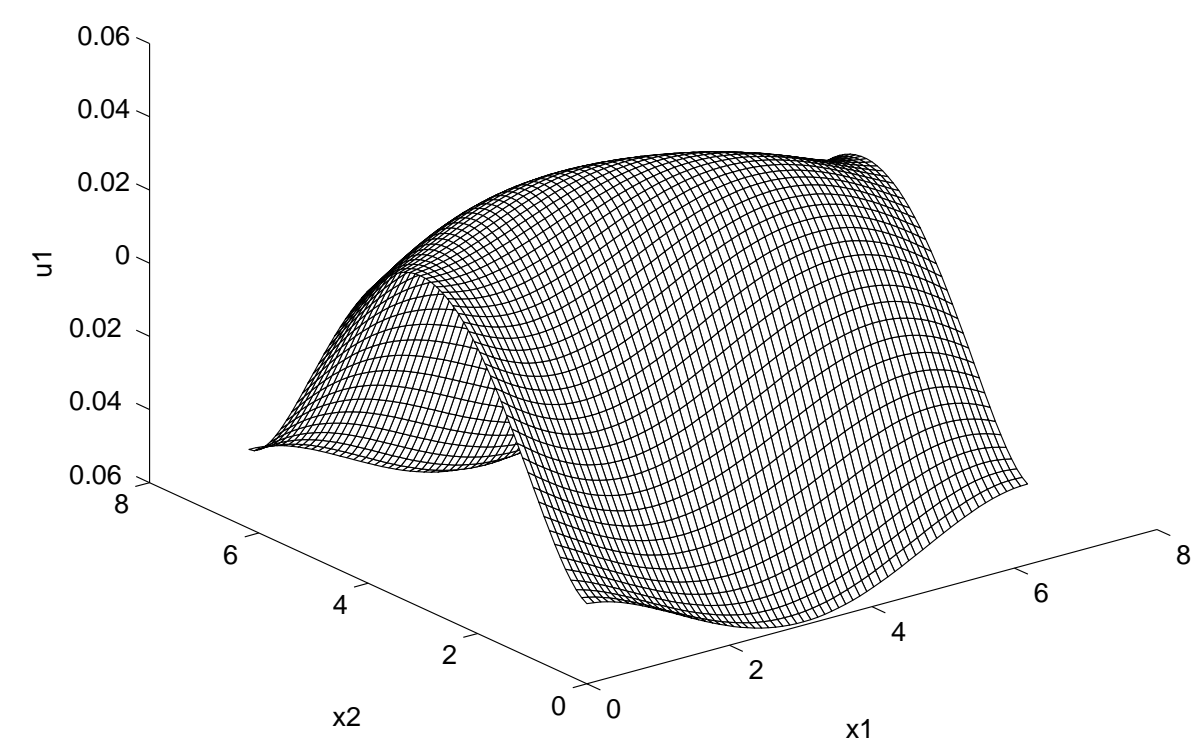

Figure 10. First component of the solution 3 at $T=4$

We have performed several numerical tests, but, for the sake of brevity, here we only present the ones related to the solution defined by (see Figure10)

$$
\begin{aligned}
& u_{0}\left(x_{1}, x_{2}\right)=P_{\text {div }}\left(\begin{array}{c}
2 \exp \left(-2\left(\left(x_{1}-\pi\right)^{2}+\left(x_{2}-\pi\right)^{2}\right)\right) \\
\exp \left(-\left(\left(x_{1}-\pi\right)^{2}+\left(x_{2}-\pi\right)^{2}\right)\right)
\end{array}\right) \\
& f\left(x_{1}, x_{2}\right)=P_{\text {div }}\left(\begin{array}{c}
\exp \left(-0.1\left(\left(x_{1}-\pi\right)^{2}+\left(x_{2}-\pi\right)^{2}\right)\right) \cos (\pi t / 2)^{5} \\
\exp \left(-0.1\left(\left(\left(x_{1}-\pi\right)^{2}+2\left(x_{2}-\pi\right)^{2}\right)\right) \cos (\pi t / 2)^{5}\right.
\end{array}\right) .
\end{aligned}
$$

We choose $T=4$ and $\mathrm{TOL}=0.01$. In Figure 11 the results of the multilevel method for $p=10$ are displayed.

We again define the efficiency index by

$$
\mathrm{Eff}=\frac{\|u-U\|_{T}}{\text { Est }_{T}} .
$$

Probably due to the bounds (3.9) for the stability constants $C_{i}^{S}$, here Eff depends on the solution. It has been computed for various values of TOL:

\begin{tabular}{|l||l|l|l|l|}
\hline TOL & $10^{-1}$ & $5.10^{-2}$ & $10^{-2}$ & $5.10^{-3}$ \\
\hline Eff & 0.055 & 0.051 & 0.060 & 0.091 \\
\hline
\end{tabular}



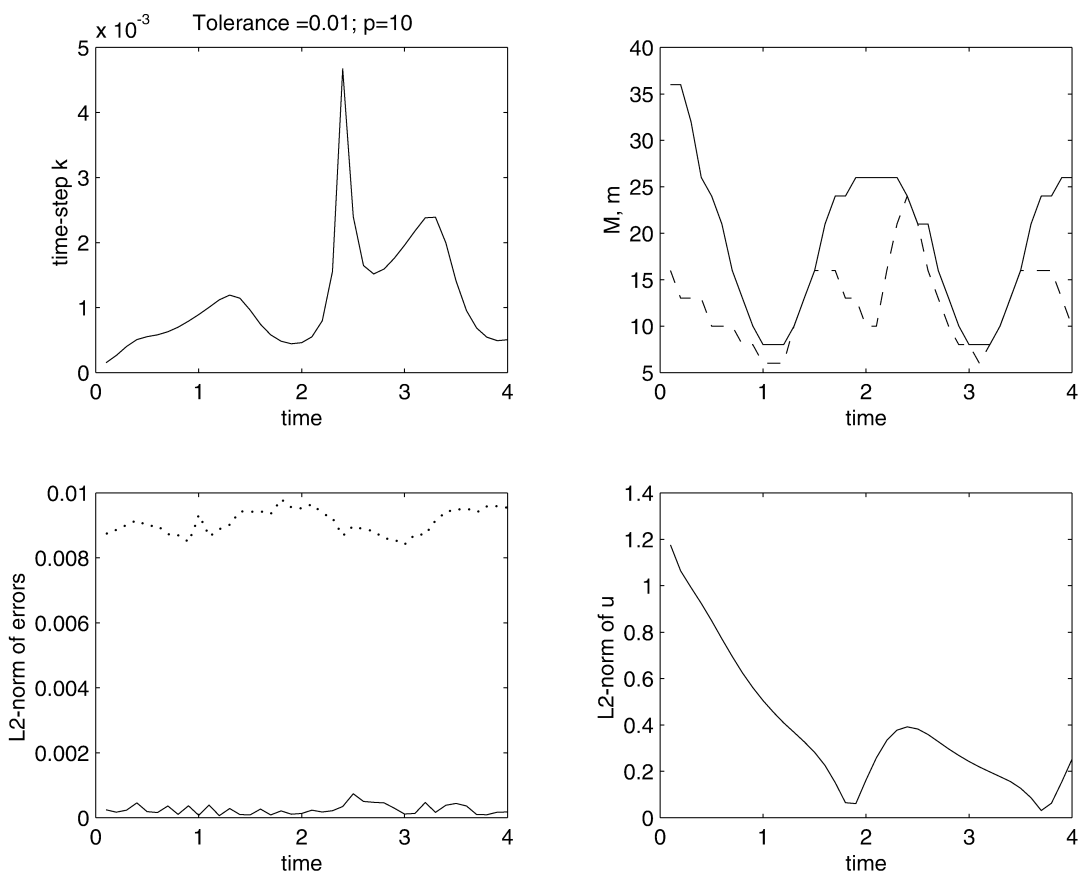

Figure 11. Solution 3 , TOL $=0.01, p=10, T=4$
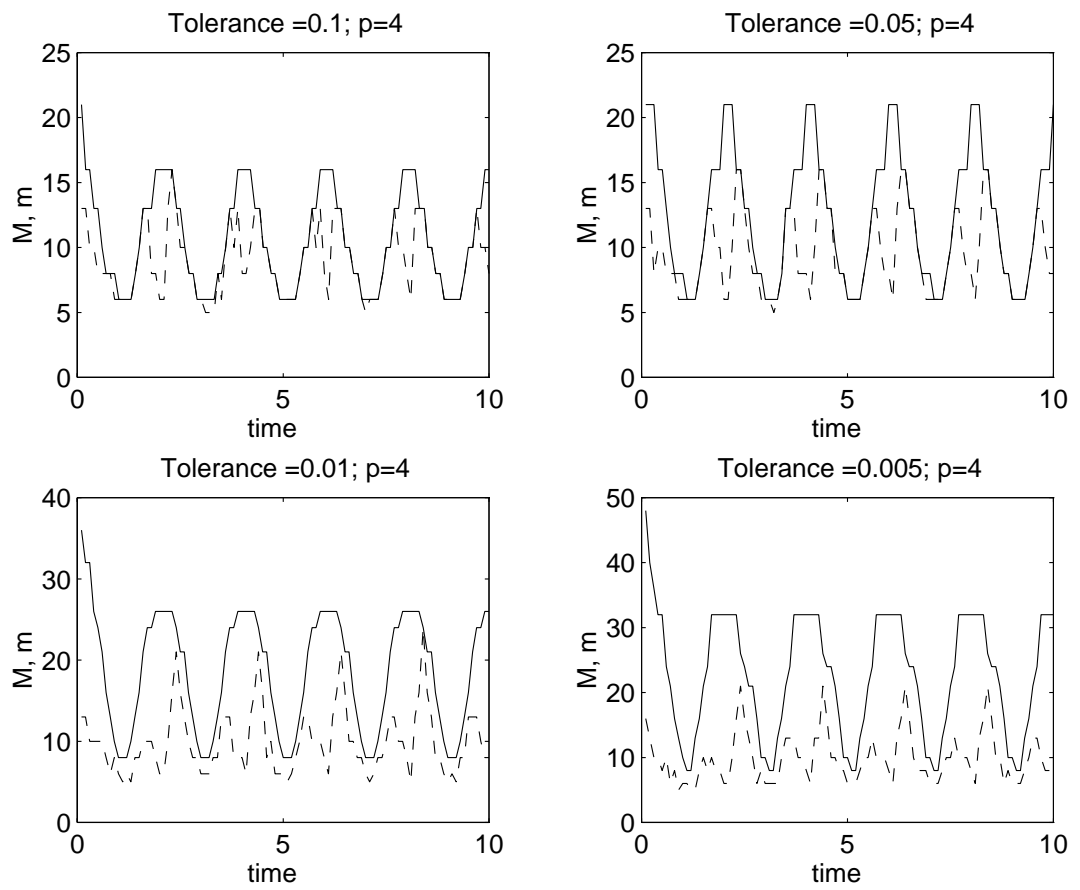

Figure 12. Solution $3, p=4, m(t)$ does not depend on TOL 
Concerning computing time, we have

\begin{tabular}{|c|c||c||c|c|c|c|c|}
\hline \multicolumn{2}{|c||}{ Solution $3(\mathrm{~T}=4)$} & 1 level & $p=4$ & $p=8$ & $p=10$ & $p=12$ & $p=16$ \\
\hline \hline \multirow{2}{*}{ TOL } & CPU time (seconds) & $190 \mathrm{~s}$ & $105 \mathrm{~s}$ & $88 \mathrm{~s}$ & $87 \mathrm{~s}$ & $88 \mathrm{~s}$ & $91 \mathrm{~s}$ \\
\cline { 2 - 8 }$=0.01$ & M-L time/1-L Time & 1 & 0.55 & 0.46 & 0.46 & 0.46 & 0.48 \\
\hline \hline $\begin{array}{c}\text { TOL } \\
=0.005\end{array}$ & CPU time (seconds) & $886 \mathrm{~s}$ & $377 \mathrm{~s}$ & $285 \mathrm{~s}$ & $274 \mathrm{~s}$ & $271 \mathrm{~s}$ & $270 \mathrm{~s}$ \\
\cline { 2 - 8 }$=$ & M-L time/1-L Time & 1 & 0.43 & 0.32 & 0.31 & 0.31 & 0.30 \\
\hline
\end{tabular}

For this solution, the gain in computing time reaches $70 \%$, and again increases as TOL decreases. Indeed, as shown on Figure 12, and as in the linear case, $p$ being a constant, there exists a function $m c(t)$, which does not depend on TOL, such that if $M(t) \geq m c(t)$ then $m(t) \simeq m c(t))$.

Therefore, compared with the one-level algorithm, the smaller TOL is, the more modes are integrated with a time step $p k>k$, and the greater the gain is.

This behaviour can be heuristically justified with an argument similar to the one given in the linear case. The details are omitted.

\section{APPENDIX}

A.1. Proof of Theorem 3.1. Following the general approach developed by C. Johnson and his coworkers (see in particular [7, 10]), the main steps of the proof consist in:

1. introducing variational formulations of the scheme (3.5) and of the linearized dual problem (3.6);

2. obtaining an error representation thanks to an appropriate solution $\varphi$ of problem (3.6);

3. using Galerkin orthogonality to condense this representation; and

4. combining interpolation error estimates and the strong stability constants $C_{i}^{S}$ defined in (3.8).

We aim to derive (3.10), that is, to estimate the error $e=u-U$ at time $t_{N}$. Recall that the continuous solution $u$ belongs to the space $\mathcal{E}$ defined by (3.4), while the approximate solution $U$ lies in $\mathcal{E}_{h}$ given by (2.3) supplemented with the divergence-free condition.

We will need some additional notation. The scalar products in $H$ and $V$ are respectively denoted by $(.,$.$) and ((.,)$.$) . Also, the error e=u-U$ lies in the following space $\mathcal{D}$ that contains both $\mathcal{E}_{h}$ and $\mathcal{E}$ :

$$
\begin{aligned}
\mathcal{D}=\left\{v \in L^{\infty}(0, T ; V)\right. & \cap L^{2}(0, T ; D(A)) \\
\forall n & \left.\in\{1, \ldots, N\}, v_{t} \in L^{2}\left(I_{n} ; H\right) \text { and } v_{n-1}^{+}, v_{n}^{-} \text {exist }\right\}
\end{aligned}
$$

where we have set

$$
v_{n-1}^{+}=\lim _{s \rightarrow 0^{+}} v\left(t_{n-1}+s\right), \quad v_{n}^{-}=\lim _{s \rightarrow 0^{-}} v\left(t_{n}+s\right), \quad\left[v_{n}\right]=v_{n}^{+}-v_{n}^{-} .
$$

We now introduce variational formulations of (3.5) and (3.6). It is easy to check that the approximate solution $U$ given by (3.5) satisfies

$$
C_{h}(U, v)=F(v), \forall v \in \mathcal{E}_{h},
$$


where $F$ is given by

$$
F(v)=\sum_{n=1}^{N} \int_{I_{n}}(f, v) d t+\left(u_{0}, v_{0}^{+}\right)
$$

and $C_{h}$ is defined by

$$
C_{h}(U, v)=\sum_{n=1}^{N} \int_{I_{n}}\left\{((U, v))+\left(B\left(U_{n-1}\right), P_{M_{n}} v\right)\right\} d t+\sum_{n=1}^{N-1}\left(\left[U_{n}\right], v_{n}^{+}\right)+\left(U_{0}^{+}, v_{0}^{+}\right),
$$

where $v \in \mathcal{D}$, and we agree to set $U_{0}=u_{0}$ for all $U \in \mathcal{E}_{h}$.

Next, the dual linearized problem (3.6) runs backward in time. Introducing the operator

$$
\begin{aligned}
L_{h}(u, v ; z, w)= & \sum_{n=1}^{N} \int_{I_{n}}\left\{\left(-w_{t}, z\right)+((w, z))+B^{*}(u, v ; z, w)\right\} d t \\
& -\sum_{n=1}^{N-1}\left(\left[w_{n}\right], z_{n}^{-}\right)+\left(w_{N}^{-}, z_{N}^{-}\right), \quad u, v, w, z \in \mathcal{D},
\end{aligned}
$$

the solution $\varphi \in \mathcal{E}$ of (3.6) with final condition $\varphi_{T} \in V$ satisfies

$$
L_{h}(u, U ; \psi, \varphi)=\left(\varphi_{T}, \psi_{N}^{-}\right), \forall \psi \in \mathcal{D} .
$$

In order to obtain a representation formula of the error $e$ at time $t_{N}$, we consider the solution $\varphi$ of (3.6) with final condition $\varphi_{T}=e_{N}^{-}=u\left(t_{N}\right)-U_{N} \in V$, and set $\psi=e$ in A.6. Then, (A.7) reads

$$
\left|u\left(t_{N}\right)-U_{N}\right|^{2}=L_{h}(u, U ; e, \varphi) .
$$

We now seek to express $L_{h}(u, U ; e, \varphi)$ in terms of the operator $C_{h}$. Let us introduce the intermediate operator $A_{h}$ defined, for $v, w \in \mathcal{D}$, by

$$
A_{h}(v, w)=\sum_{n=1}^{N} \int_{I_{n}}\left\{\left(v_{t}, w\right)+((v, w))+(B(v), w)\right\} d t+\sum_{n=1}^{N-1}\left(\left[v_{n}\right], w_{n}^{+}\right)+\left(v_{0}^{+}, w_{0}^{+}\right) .
$$

Then, by integrating by parts both in space and time, it is easy to check the following identity:

$$
\forall u, v, w \in \mathcal{D}, L_{h}(u, v ; u-v, w)=A_{h}(u, w)-A_{h}(v, w) .
$$

Therefore, A.8 can be written as

$$
\left|u\left(t_{N}\right)-U_{N}\right|^{2}=A_{h}(u, \varphi)-A_{h}(U, \varphi)
$$

Besides, if $u \in \mathcal{E}$ is the solution of the continuous problem, the following identity is satisfied:

$$
A_{h}(u, v)=F(v), \quad \forall v \in \mathcal{E},
$$

so that A.11 becomes

$$
\left|u\left(t_{N}\right)-U_{N}\right|^{2}=F(\varphi)-A_{h}(U, \varphi) .
$$


Finally, recalling the definition (A.5), we find that

$$
\begin{aligned}
\left|u\left(t_{N}\right)-U_{N}\right|^{2}= & F(\varphi)-C_{h}(U, \varphi) \\
& +\sum_{n=1}^{N} \int_{I_{n}}\left\{\left(B\left(U_{n-1}\right)-B\left(U_{n}\right), P_{M_{n}} \varphi\right)-\left(B\left(U_{n}\right), Q_{M_{n}} \varphi\right)\right\} d t .
\end{aligned}
$$

Next, let $\Psi \in \mathcal{E}_{h}$. Thanks to the variational formulation A.3 of the scheme (3.5), we obtain the following error representation formula:

$$
\begin{aligned}
\left|u\left(t_{N}\right)-U_{N}\right|^{2}= & F(\varphi-\Psi)-C_{h}(U, \varphi-\Psi) \\
& +\sum_{n=1}^{N} \int_{I_{n}}\left\{\left(B\left(U_{n-1}\right)-B\left(U_{n}\right), P_{M_{n}} \varphi\right)-\left(B\left(U_{n}\right), Q_{M_{n}} \varphi\right)\right\} d t,
\end{aligned}
$$

which we expand by replacing $F$ and $C_{h}$ with their expressions (A.4) and (A.5), to conclude that

$$
\begin{aligned}
\mid u\left(t_{N}\right) & -\left.U_{N}\right|^{2}=\sum_{n=1}^{N} \int_{I_{n}}\left\{(f, \varphi-\Psi)-((U, \varphi-\Psi))-\left(B\left(U_{n-1}\right), P_{M_{n}}(\varphi-\Psi)\right)\right\} d t \\
& -\sum_{n=0}^{N-1}\left(\left[U_{n}\right],(\varphi-\Psi)_{n}^{+}\right) \\
& +\sum_{n=1}^{N} \int_{I_{n}}\left\{\left(B\left(U_{n-1}\right)-B\left(U_{n}\right), P_{M_{n}} \varphi\right)-\left(B\left(U_{n}\right), Q_{M_{n}} \varphi\right)\right\} d t .
\end{aligned}
$$

Now we take $\Psi=\widetilde{\varphi}$ in A.14 , where $\widetilde{\varphi}$ is the orthogonal projection of $\varphi$ on $\mathcal{E}_{h}$, that is,

$$
\left.\widetilde{\varphi}(t)\right|_{I_{n}}=P_{M n} \pi_{n} \varphi(t)=\pi_{n} P_{M n} \varphi(t), \text { with } \pi_{n} \varphi=\frac{1}{k_{n}} \int_{I_{n}} \varphi(., t) d t .
$$

Due to the Galerkin orthogonality properties

$$
\int_{I_{n}}((U, \varphi-\widetilde{\varphi})) d t=\int_{I_{n}}\left(\left(U, \varphi-\pi_{n} \varphi\right)\right) d t+\int_{I_{n}}\left(\left(U, Q_{M_{n}} \pi_{n} \varphi\right)\right) d t=0,
$$

(A.14) reduces to

$$
\begin{aligned}
& \left|u\left(t_{N}\right)-U_{N}\right|^{2}=\sum_{n=1}^{N} \int_{I_{n}}(f, \varphi-\widetilde{\varphi}) d t-\sum_{n=0}^{N-1}\left(\left[U_{n}\right],(\varphi-\widetilde{\varphi})_{n}^{+}\right) \\
& \quad+\sum_{n=1}^{N} \int_{I_{n}}\left(B\left(U_{n-1}\right)-B\left(U_{n}\right), P_{M_{n}} \varphi\right) d t-\sum_{n=1}^{N} \int_{I_{n}}\left(B\left(U_{n}\right), Q_{M_{n}} \varphi\right) d t .
\end{aligned}
$$

Next, we aim to majorize the right-hand side of A.15. For that purpose, it is convenient to write

$$
\left.(\varphi-\widetilde{\varphi})\right|_{I_{n}}=\left(\varphi-P_{M_{n}} \varphi\right)+\left(P_{M_{n}} \varphi-P_{M_{n}} \pi_{n} \varphi\right),
$$


so that A.15 becomes

(A.16)

$$
\begin{aligned}
\mid u\left(t_{N}\right) & -\left.U_{N}\right|^{2} \\
= & \sum_{n=1}^{N} \int_{I_{n}}\left(f, P_{M_{n}} \varphi-\pi_{n} P_{M_{n}} \varphi\right) d t-\sum_{n=1}^{N}\left(\left[U_{n-1}\right], P_{M_{n}} \varphi_{n-1}^{+}-\pi_{n} P_{M_{n}} \varphi\right) \\
& +\sum_{n=1}^{N} \int_{I_{n}}\left(f, \varphi-P_{M_{n}} \varphi\right) d t-\sum_{n=1}^{N}\left(\left[U_{n-1}\right], \varphi_{n-1}^{+}-P_{M_{n}} \varphi_{n-1}^{+}\right) \\
& +\sum_{n=1}^{N} \int_{I_{n}}\left(B\left(U_{n-1}\right)-B\left(U_{n}\right), P_{M_{n}} \varphi\right) d t-\sum_{n=1}^{N} \int_{I_{n}}\left(B\left(U_{n}\right), Q_{M_{n}} \varphi\right) d t \\
= & \mathrm{I}+\mathrm{II}+\mathrm{III}+\mathrm{IV}+\mathrm{V}+\mathrm{VI} .
\end{aligned}
$$

The terms in the right-hand side of (A.16) have been denoted by I-VI, and we intend to bound them, thanks to the following interpolation estimates for $P_{M}$ and $\pi_{n}$ :

$$
\begin{gathered}
\left|u-P_{M} u\right| \leq 4 M^{-2}|A u|, \\
\left\|u-\pi_{n} u\right\|_{I_{n}} \leq \min \left(2\|u\|_{I_{n}}, \int_{I_{n}}\left|u_{t}\right| d t\right),
\end{gathered}
$$

together with the stability constants $C_{i}^{S}$ defined in (3.8).

Beginning with the first term, we find that

$$
\begin{aligned}
|\mathrm{I}| & =\left|\sum_{n=1}^{N} \int_{I_{n}}\left(f-\pi_{n} f, P_{M_{n}} \varphi-\pi_{n} P_{M_{n}} \varphi\right) d t\right| \\
& \leq \sum_{n=1}^{N} \int_{I_{n}}\left|f-\pi_{n} f\right|\left|\varphi-\pi_{n} \varphi\right| d t \\
& \leq\left\|k^{2} f_{t}\right\|_{T}\left(2\|\varphi\|_{I_{N}}+\int_{0}^{T-k_{N}}\left|\varphi_{t}\right| d t\right) .
\end{aligned}
$$

Therefore, due to the definition (3.8 $)$ for $C_{1}^{S}$, we infer that

$$
|\mathrm{I}| \leq\left(2+L_{N}\right) C_{1}^{S}(T, u, U)\left\|k^{2} f_{t}\right\|_{T}\left|u\left(t_{N}\right)-U_{N}\right| .
$$

Similarly, it can be shown that

$$
|\mathrm{II}| \leq\left(2+L_{N}\right) C_{1}^{S}(T, u, U) \max _{n \in\{1, \ldots, N\}}\left|\left[U_{n-1}\right]\right|\left|u\left(t_{N}\right)-U_{N}\right| .
$$

Here, as $\left.U\right|_{I_{n}}=U_{n}$, we have $\left[U_{n-1}\right]=U_{n}-U_{n-1}$.

The estimate of the third term is more involved. We decompose it as follows:

$$
\mathrm{III}=\sum_{n=1}^{N-1} \int_{I_{n}}\left(Q_{M_{n}} f, Q_{M_{n}} \varphi\right) d t+\int_{I_{N}}\left(Q_{M_{N}} f, Q_{M_{N}} \varphi\right) d t=\mathrm{IIIa}+\mathrm{IIIb}
$$

The first term in (A.21) satisfies

$$
\begin{aligned}
\mid \text { IIIa } \mid & \leq 4\left\|M^{-2} Q_{M} f\right\|_{T} \int_{0}^{t_{N-1}}|A \varphi| d t \\
& \leq 4 L_{N} C_{2}^{S}(T, u, U)\left\|M^{-2} Q_{M} f\right\|_{T}\left|u\left(t_{N}\right)-U_{N}\right| .
\end{aligned}
$$


Next, in order to estimate IIIb, we consider

$$
\Phi(t)=\int_{T}^{t} \varphi(s) d s
$$

and, by integrating by parts, we find that

$$
\mathrm{IIIb}=-\left(Q_{M_{N}} f\left(t_{N-1}\right), Q_{M_{N}} \Phi\left(t_{N-1}\right)\right)-\int_{I_{N}}\left(Q_{M_{N}} f_{t}, Q_{M_{N}} \Phi\right) d t .
$$

Then, on one hand, we note that

$$
\begin{aligned}
\left|\left(Q_{M_{N}} f\left(t_{N-1}\right), Q_{M_{N}} \Phi\left(t_{N-1}\right)\right)\right| & \leq 4\left\|M^{-2} Q_{M} f\right\|_{I_{N}}\left|A \Phi\left(t_{N-1}\right)\right| \\
& \leq 4\left\|M^{-2} Q_{M} f\right\|_{I_{N}}\left|\int_{T}^{t_{N-1}} A \varphi(s) d s\right|,
\end{aligned}
$$

which implies by $(\underline{3.8 \mathrm{c}})$ that

$$
\left|\left(Q_{M_{N}} f\left(t_{N-1}\right), Q_{M_{N}} \Phi\left(t_{N-1}\right)\right)\right| \leq 4 C_{3}^{S}(T, u, U)\left\|M^{-2} Q_{M} f\right\|_{I_{N}}\left|u\left(t_{N}\right)-U_{N}\right| .
$$

On the other hand, we similarly write

$$
\begin{aligned}
\left|\int_{I_{N}}\left(Q_{M_{N}} f_{t}, Q_{M_{N}} \Phi\right) d t\right| & \leq 4 k_{N} M_{N}^{-2}\|A \Phi\|_{I_{N}}\left\|Q_{M_{N}} f_{t}\right\|_{I_{N}} \\
& \leq 4 k_{N} M_{N}^{-2}\left\|\int_{t}^{T} A \varphi(s) d s\right\|_{I_{N}}\left\|Q_{M} f_{t}\right\|_{I_{N}} \\
& \leq 4 C_{3}^{S}(T, u, U)\left\|k M^{-2} Q_{M} f_{t}\right\|_{I_{N}}\left|u\left(t_{N}\right)-U_{N}\right| .
\end{aligned}
$$

Next, since $Q_{M_{n}}\left[U_{n-1}\right]=-Q_{M_{n}} U_{n-1}$, the fourth term is such that

$$
|\mathrm{IV}| \leq \sum_{n=1}^{N} 4\left|\frac{Q_{M_{n}} U_{n-1}}{k_{n} M_{n}^{2}}\right| k_{n}\left|A \varphi\left(t_{n-1}\right)\right|,
$$

which due to (3.8 d) for $C_{4}^{S}$ yields

$$
|\mathrm{IV}| \leq 4 L_{N} C_{4}^{S}(T, u, U) \max _{n \in\{1, \ldots, N\}}\left|\frac{Q_{M_{n}} U_{n-1}}{k_{n} M_{n}^{2}}\right|\left|u\left(t_{N}\right)-U_{N}\right| .
$$

Thanks to (3.8) we obtain

$$
|\mathrm{V}| \leq C_{5}^{S}(T, u, U) \max _{n \in\{1, \ldots, N\}}\left|P_{M_{n}}\left(B\left(U_{n}\right)-B\left(U_{n-1}\right)\right)\right|\left|u\left(t_{N}\right)-U_{N}\right| .
$$

Finally, the sixth term splits analogously to A.21 and one can check that

$$
\begin{aligned}
& \left|\sum_{n=1}^{N-1} \int_{I_{n}}\left(B\left(U_{n}\right), Q_{M_{n}} \varphi\right) d t\right| \\
& \quad \leq 4 L_{N} C_{2}^{S}(T, u, U) \max _{n \in\{1, \ldots, N\}}\left|M_{n}^{-2} Q_{M_{n}} B\left(U_{n}\right)\right|\left|u\left(t_{N}\right)-U_{N}\right| \\
& \left|\int_{I_{N}}\left(B\left(U_{N}\right), Q_{M_{N}} \varphi\right) d t\right| \leq 4 C_{3}^{S}(T, u, U)\left|M_{N}^{-2} Q_{M_{N}} B\left(U_{N}\right)\right|\left|u\left(t_{N}\right)-U_{N}\right| .
\end{aligned}
$$


Combining (A.15) with (A.19), (A.20), (A.26), (A.22), (A.24), (A.25), A.27), (A.28) and (A.29) provides the bound (3.10), and concludes the proof of Theorem 3.1.

A.2. Proof of Theorem 3.2. The proof follows steps analogous to the ones above for Theorem 3.1

We aim to estimate the error $e=u-U$ at time $t_{N}$. Here, the approximate solution given by the scheme (3.12) belongs to the space $\mathcal{F}_{h}$ defined by (2.10) supplemented with the divergence-free condition. Since $\mathcal{F}_{h} \subset \mathcal{D}$, the error $e$ still belongs to $\mathcal{D}$ given by (A.1).

First, we introduce a variational formulation for the scheme (3.12). The approximate solution $U$ satisfies

$$
D_{h}(U, v)=F(v), \forall v \in \mathcal{F}_{h},
$$

where $F$ is given by A.4 and $D_{h}$ by

$$
\begin{aligned}
D_{h}(U, v) & =\sum_{n=1}^{N} \int_{I_{n}}((U, v)) d t+\sum_{n=1}^{N} \int_{I_{n}}\left(B\left(U_{n-1}\right), P_{m_{n}} v\right) d t \\
+ & \sum_{q=1}^{Q} \int_{J_{q}}\left(B\left(U_{n_{q}-1}\right),\left(P_{M_{q}}-P_{m_{q}}\right) v\right)+\sum_{n=1}^{N-1}\left(\left[U_{n}\right], v_{n}^{+}\right)+\left(U_{0}^{+}, v_{0}^{+}\right),
\end{aligned}
$$

where we have set $U_{0}=u_{0}$ for all $U \in \mathcal{F}_{h}$.

The second step is quite similar to the one-level case. We again consider a solution $\varphi$ of (3.6) with $\varphi_{T}=e_{N}^{-}$. Then the equalities (A.8) (A.13) still hold, and therefore we again have

$$
\left|u\left(t_{N}\right)-U_{N}\right|^{2}=F(\varphi)-A_{h}(U, \varphi)
$$

where the operator $A_{h}$ is defined by A.9. Making use of $D_{h}$, equality A.32 becomes

$$
\begin{aligned}
\left|u\left(t_{N}\right)-U_{N}\right|^{2}= & F(\varphi)-D_{h}(U, \varphi)+\sum_{n=1}^{N} \int_{I_{n}}\left(B\left(U_{n-1}\right), P_{m_{n}} \varphi\right) d t \\
& +\sum_{q=1}^{Q} \int_{J_{q}}\left(B\left(U_{n_{q}-1}\right),\left(P_{M_{q}}-P_{m_{q}}\right) \varphi\right) d t-\sum_{n=1}^{N} \int_{I_{n}}\left(B\left(U_{n}\right), \varphi\right) d t .
\end{aligned}
$$

This equality also can be rewritten as

$$
\begin{aligned}
& \left|u\left(t_{N}\right)-U_{N}\right|^{2}=F(\varphi)-D_{h}(U, \varphi)+\sum_{n=1}^{N} \int_{I_{n}}\left(B\left(U_{n-1}\right)-B\left(U_{n}\right), P_{m_{n}} \varphi\right) d t \\
& +\sum_{q=1}^{Q} \int_{J_{q}}\left(B\left(U_{n_{q}-1}\right)-B\left(U_{n}\right),\left(P_{M_{q}}-P_{m_{q}}\right) \varphi\right) d t-\sum_{n=1}^{N} \int_{I_{n}}\left(B\left(U_{n}\right), Q_{M_{n}} \varphi\right) d t .
\end{aligned}
$$


Let $\Psi$ be any element of $\mathcal{F}_{h}$. Thanks to the variational formulation A.30 of the scheme (3.12), we obtain

$$
\begin{gathered}
\left|u\left(t_{N}\right)-U_{N}\right|^{2}=F(\varphi-\Psi)-D_{h}(U, \varphi-\Psi)+\sum_{n=1}^{N} \int_{I_{n}}\left(B\left(U_{n-1}\right)-B\left(U_{n}\right), P_{m_{n}} \varphi\right) d t \\
+\sum_{q=1}^{Q} \int_{J_{q}}\left(B\left(U_{n_{q}-1}\right)-B(U),\left(P_{M_{q}}-P_{m_{q}}\right) \varphi\right) d t-\sum_{n=1}^{N} \int_{I_{n}}\left(B(U), Q_{M_{n}} \varphi\right) d t .
\end{gathered}
$$

We expand this last equality by replacing $F$ and $D_{h}$ with their expressions. Then, for all $\Psi \in \mathcal{F}_{h}$, the following error representation formula holds:

$$
\begin{aligned}
\mid u\left(t_{N}\right) & -\left.U_{N}\right|^{2}=\sum_{n=1}^{N} \int_{I_{n}}\{(f, \varphi-\Psi)-((U, \varphi-\Psi))\} d t \\
& -\sum_{n=1}^{N} \int_{I_{n}}\left(B\left(U_{n-1}\right), P_{m_{n}}(\varphi-\Psi)\right) d t \\
& -\sum_{q=1}^{Q} \int_{J_{q}}\left(B\left(U_{n_{q}-1}\right),\left(P_{M_{q}}-P_{m_{q}}\right)(\varphi-\Psi)\right) d t \\
& -\sum_{n=0}^{N-1}\left(\left[U_{n}\right],(\varphi-\Psi)_{n}^{+}\right)+\sum_{n=1}^{N} \int_{I_{n}}\left(B\left(U_{n-1}\right)-B\left(U_{n}\right), P_{m_{n}} \varphi\right) d t \\
& +\sum_{q=1}^{Q} \int_{J_{q}}\left(B\left(U_{n_{q}-1}\right)-B(U),\left(P_{M_{q}}-P_{m_{q}}\right) \varphi\right) d t \\
& -\sum_{n=1}^{N} \int_{I_{n}}\left(B(U), Q_{M_{n}} \varphi\right) d t .
\end{aligned}
$$

Now we take $\Psi=\widehat{\varphi}$ in (A.34), where $\widehat{\varphi}$ is the orthogonal projection of $\varphi$ on $\mathcal{F}_{h}$, that is,

$$
\left.\widehat{\varphi}(t)\right|_{I_{n}}=P_{m_{q}} \pi_{n} \varphi+\left(P_{M_{q}}-P_{m_{q}}\right) \pi_{q} \varphi, \text { for } I_{n} \subset J_{q},
$$

where

$$
\pi_{n} \varphi=\frac{1}{k_{n}} \int_{I_{n}} \varphi(., t) d t, \quad \pi_{q} \varphi=\frac{1}{K_{q}} \int_{J_{q}} \varphi(., t) d t
$$

Using Galerkin orthogonality properties such as

$$
-\sum_{n=1}^{N} \int_{I_{n}}\left(B\left(U_{n-1}\right), P_{m_{n}}(\varphi-\widehat{\varphi})\right) d t-\sum_{q=1}^{Q} \int_{J_{q}}\left(B\left(U_{n_{q}-1}\right),\left(P_{M_{q}}-P_{m_{q}}\right)(\varphi-\widehat{\varphi})\right)=0
$$


we see that

$$
\begin{aligned}
\mid u\left(t_{N}\right) & -\left.U_{N}\right|^{2}=\sum_{n=1}^{N} \int_{I_{n}}(f, \varphi-\widehat{\varphi}) d t-\sum_{n=0}^{N-1}\left(\left[U_{n}\right],(\varphi-\widehat{\varphi})_{n}^{+}\right) \\
& +\sum_{n=1}^{N} \int_{I_{n}}\left(B\left(U_{n-1}\right)-B\left(U_{n}\right), P_{m_{n}} \varphi\right) d t \\
& +\sum_{q=1}^{Q} \int_{J_{q}}\left(B\left(U_{n_{q}-1}\right)-B(U),\left(P_{M_{q}}-P_{m_{q}}\right) \varphi\right) d t-\sum_{n=1}^{N} \int_{I_{n}}\left(B(U), Q_{M_{n}} \varphi\right) d t .
\end{aligned}
$$

Next, writing

$$
\left.(\varphi-\widehat{\varphi})\right|_{I_{n}}=P_{m_{n}}\left(I-\pi_{n}\right) \varphi+\left(P_{M_{q}}-P_{m_{q}}\right)\left(I-\pi_{q}\right) \varphi+\left(I-P_{M_{q}}\right) \varphi
$$

and using that fact that, for all $n \neq n_{q}$ such that $I_{n} \subset J_{q}$,

$$
\left(P_{M_{q}}-P_{m_{q}}\right)\left[U_{n-1}\right]=\left(I-P_{M_{q}}\right)\left[U_{n-1}\right]=0,
$$

(A.35) becomes

$$
\begin{aligned}
\left|u\left(t_{N}\right)-U_{N}\right|^{2} & =\sum_{n=1}^{N} \int_{I_{n}}\left(f, P_{m_{n}}\left(I-\pi_{n}\right) \varphi\right) d t-\sum_{n=1}^{N}\left(\left[U_{n-1}\right], P_{m_{n}} \varphi_{n-1}^{+}-\pi_{n} P_{m_{n}} \varphi\right) \\
& +\sum_{n=1}^{N} \int_{I_{n}}\left(f, \varphi-P_{M_{n}} \varphi\right) d t-\sum_{q=1}^{Q}\left(\left[U_{n_{q}-1}\right], \varphi_{n_{q}-1}^{+}-P_{M_{q}} \varphi_{n_{q}-1}^{+}\right) \\
& +\sum_{n=1}^{N} \int_{I_{n}}\left(B\left(U_{n-1}\right)-B\left(U_{n}\right), P_{m_{n}} \varphi\right) d t-\sum_{n=1}^{N} \int_{I_{n}}\left(B\left(U_{n}\right), Q_{M_{n}} \varphi\right) d t \\
& +\sum_{q=1}^{Q} \int_{J_{q}}\left(f,\left(P_{M_{q}}-P_{m_{q}}\right)\left(I-\pi_{q}\right) \varphi\right) d t \\
& -\sum_{q=1}^{Q}\left(\left[U_{n_{q}-1}\right],\left(P_{M_{q}}-P_{m_{q}}\right)\left(\varphi-\pi_{q} \varphi\right)_{n_{q}-1}^{+}\right) \\
& +\sum_{q=1}^{Q} \int_{J_{q}}\left(B\left(U_{n_{q}-1}\right)-B(U),\left(P_{M_{q}}-P_{m_{q}}\right) \varphi\right) d t \\
= & \mathrm{I}+\mathrm{II}+\mathrm{III}+\mathrm{IV}+\mathrm{V}+\mathrm{VI}+\mathrm{VII}+\mathrm{VIII}+\mathrm{IX} .
\end{aligned}
$$

We now use the interpolation estimates (A.17) and (A.18) and the corresponding ones for $P_{m}$ and $\pi_{q}$ together with the stability constants $C_{i}^{S}$ defined in (3.8) to majorize the terms I-IX in (A.36). Actually, the terms I-VI are similar to the previous ones in (A.16), and computations as above yield the first five lines of the a posteriori estimate (3.13). 
Next, we have:

$$
\begin{aligned}
|\mathrm{VII}| & =\left|\sum_{q=1}^{Q} \int_{J_{q}}\left(\left(I-\pi_{q}\right)\left(P_{M_{q}}-P_{m_{q}}\right) f, \varphi-\pi_{q} \varphi\right) d t\right| \\
& \leq\left\|K^{2}\left(P_{M}-P_{m}\right) f_{t}\right\|_{T}\left(2\|\varphi\|_{J_{Q}}+\int_{0}^{T-K_{Q}}\left|\varphi_{t}\right| d t\right) .
\end{aligned}
$$

Therefore, recalling the definition (3.8 $)$ of $C_{1}^{S}$, since $k_{N} \leq K_{Q}$, which implies $\sqrt{\left|\log K_{Q}\right|} \leq L_{N}$ (assuming $K_{Q} \leq 1$ ), we infer the following bound:

$$
|\mathrm{VII}| \leq\left(2+L_{N}\right) C_{1}^{S}(T, u, U)\left\|K^{2}\left(P_{M}-P_{m}\right) f_{t}\right\|_{T}\left|u\left(t_{N}\right)-U_{N}\right| .
$$

Similarly, it can be checked that

$$
|\mathrm{VIII}| \leq\left(2+L_{N}\right) C_{1}^{S}(T, u, U) \max _{q \in\{1, \ldots, Q\}}\left|\left(P_{M_{q}}-P_{m_{q}}\right)\left(U_{n_{q}}-U_{n_{q}-1}\right)\right|\left|u\left(t_{N}\right)-U_{N}\right| .
$$

Finally, for the last term we have

$$
\begin{aligned}
& \left|\sum_{q=1}^{Q} \int_{J_{q}}\left(B\left(U_{n_{q}-1}\right)-B(U),\left(P_{M_{q}}-P_{m_{q}}\right) \varphi\right) d t\right| \\
& \leq C_{5}^{S}(T, u, U) \max _{q}\left\{\max _{n \text { s.t. } I_{n} \subset J_{q}}\left|\left(P_{M_{q}}-P_{m_{q}}\right)\left(B\left(U_{n}\right)-B\left(U_{n_{q}-1}\right)\right)\right|\right\}\left|u\left(t_{N}\right)-U_{N}\right| .
\end{aligned}
$$

Combining these estimates with (A.36) provides (3.13), and concludes the proof of Theorem 3.2.

\section{REFERENCES}

1. J.-B. Burie, Thèse, Ecole Centrale de Lyon, 1996.

2. J.-B. Burie and M. Marion, Multilevel methods in space and time for the Navier-Stokes equations, SIAM J. Numer. Anal., 34 (1997), pp. 1574-1599. MR 98h:65047

3. C. Canuto, M. Y. Hussaini, A. Quarteroni and T. A. Zang, Spectral Methods in Fluid Dynamics, Springer-Verlag, New York-Heidelberg-Berlin, 1987. MR 89m:76004

4. A. Debussche, T. Dubois and R. Temam, The nonlinear Galerkin method: A multi-scale method applied to the simulation of homogenous turbulent flows, ICASE Report No 93-93 (1993).

5. K. Eriksson and C. Johnson, Adaptive finite element methods for parabolic problems, I: A linear model problem, SIAM J. Numer. Anal., 28 (1991), pp. 43-77. MR 91m:65274

6. K. Eriksson and C. Johnson, Adaptive finite element methods for parabolic problems, II: Optimal error estimates in $L_{\infty}\left(L_{2}\right)$ and $L_{\infty}\left(L_{\infty}\right)$, SIAM J. Numer. Anal., 32 (1995), pp. $706-$ 740. MR 96c:65162

7. K. Eriksson and C. Johnson, Adaptive finite element methods for parabolic problems, IV: Nonlinear problems, SIAM J. Numer. Anal., 32 (1995), pp. 1729-1749. MR 96i:65081

8. K. Eriksson, C. Johnson and V. Thomée, Time discretization of parabolic problems by the Discontinuous Galerkin method, RAIRO Modél Math. Anal. Numér. 19 (1985), pp. 611-643. MR 87e:65073

9. C. Foias, O. Manley and R. Temam, Modelling of the interaction of small and large eddies in two-dimensional turbulent flows, RAIRO Modél Math. Anal. Numér. 22 (1988), pp. 93-118. MR 89h:76022 
10. C. Johnson, R. Rannacher and M. Boman, Numerics and hydrodynamic stability: Towards error control in CFD, SIAM J. Numer. Anal., 32 (1995), pp. 1058-1079. MR 96j:76089

11. M. Marion and J. Xu, Error estimates on a new nonlinear Galerkin method based on two-grid finite elements, SIAM J. Numer. Anal., 32 (1995), pp. 1170-1184. MR 96f:65136

UPRESA S466, Mathématiques Appliquées de Bordeaux, Université Victor Segalen Bordeaux 2, BP26, 146 rue Léo-Saignat, 33076 Bordeaux Cedex, France

E-mail address: burie@u-bordeaux2.fr

UMR CNRS 5585 et Département Mathématiques-Informatique, Ecole Centrale de Lyon, BP 163, 69131 ECULly Cedex, France

E-mail address: marion@cc.ec-lyon.fr 